\title{
Effects of Chilling Injury, Physical and Biochemical Changes on Grafted Watermelons Stored at Low Temperature
}

\section{Elif ÇANDIR ${ }^{1}$ (D) Ahmet Erhan ÖZDEMIR ${ }^{1}$ (D) Halit YETişiR ${ }^{2}$ Veysel ARAS $^{3 *}$ (1) Ömer ARSLAN ${ }^{4}$ (D) Özay BALTAER ${ }^{1}$ (D) Mustafa ÜNLÜ ${ }^{3}$}

\author{
${ }^{1}$ Hatay Mustafa Kemal University Faculty of Agriculture Department of Horticulture, 31001, Antakya, Turkey \\ ${ }^{2}$ Erciyes University Seyrani Faculty of Agriculture Department of Horticulture, 38030, Kayseri, Turkey \\ ${ }^{3}$ Alata Horticultural Research Institute, 33740, Mersin, Turkey \\ ${ }^{4}$ Mersin Directorate of Provincial Agriculture and Forestry, 33130, Mersin, Turkey
}

\section{Article History}

Received 31 March 2021

Accepted 25 May 2021

First Online 14 June 2021

\section{Corresponding Author}

E-mail: varas2001@yahoo.com

\section{Keywords}

Grafting

Quality

Rootstock

Storage

Watermelon

\begin{abstract}
Watermelon fruit from Crimson Tide (CT) and Crisby (CR) grafted onto Ferro, RS841, Argentario, and Macis rootstocks and ungrafted CT and CR were compared for effects of low temperature storage on chilling injury, physical and biochemical changes at $0^{\circ} \mathrm{C}$ and $85-90 \%$ relative humidity for 21 days. After storage, fruit were hold to $21^{\circ} \mathrm{C}$ and $75-80 \%$ relative humidity for 7 days to determine shelf life. Quality analyses were determined during storage and shelf life at a weekly interval. The chilling injury areas covered $<25 \%$ of rind surface of fruit for both cultivars. Weight loss in grafted and control fruit were very low $(<1 \%)$ during storage for both cultivars. Fungal decay was not observed during storage for both cultivars, but it was seen during the shelf life for both cultivars. Total soluble solid content remained above $10 \%$ in fruit of both cultivars throughout storage period. Lycopene content significantly decreased at the end of storage for both cultivars.
\end{abstract}

\section{Introduction}

Soil borne diseases (caused by Fusarium and Verticillium species etc.) due to continuous and intensive cultivation are limiting factors affecting early season watermelon cultivation in plastic tunnels and later in open field conditions. Lagenaria and Cucurbita rootstocks are known to be resistant to Fusarium wilt and other soil-borne diseases; it provides advantages to watermelon cultivation to control diseases, to eliminate plant rotation and to increase yield as an alternative to other disinfection methods. The primary reason for grafting watermelon has been for Fusarium resistance, but it can be used to provide resistance or increase tolerance to Phytophthora blight, Verticillium wilt, Phomopsis rot, root-knot nematodes and in some cases viruses (Davis et al., 2008). Grafting has also been effective at increasing the cold tolerance of watermelon (Miguel et al., 2004). In addition, grafting impress fruit quality such as flesh firmness, fruit pulp color, lycopene and sugar amount. There is little work on the postharvest physiology of grafted or ungrafted watermelons. Storage and shelf of watermelons is confined by low temperature $\left(<7^{\circ} \mathrm{C}\right)$ and high temperature. Fruits are susceptible to chilling injury and flesh color rotting and loss of color at lower temperatures and fruit are exposing to rotting and sugar loss at higher temperatures (Chisholm and Picha, 1986). The usual shelf life for watermelon is $14-21$ days after harvest at $13^{\circ} \mathrm{C}$ (Rushing et al., 2001). Watermelons are generally not cooled when shipped locally. But, watermelons ripen in the hot summer months and are exposed to high temperatures during marketing. Cold storage and shipping can be preferred during export shipping to extend shelf life.

In the Mediterranean basin, where agricultural land for long rotations is unavailable, use of resistant rootstocks, largely interspecific cucurbit hybrids has become imperative for watermelon production (Kyriacou and Soteriou, 2015). Reports 
on watermelon quality with respect to grafting have been conflicting, indicative of a rootstockdependant effect or a rootstock-scion interaction (Yetisir et al., 2003; Miguel et al., 2004; Davis and Perkins-Veazie, 2005; Taylor et al., 2006).

Postharvest quality of watermelon fruit from Crisby (CR) and Crimson Tide (CT) grafted onto Ferro, RS841, Argentario and Macis rootstocks and non-grafted CR and CT were determined in 21 days during the storage at $7^{\circ} \mathrm{C}$. The storage period of watermelons in good quality were determined as 21 days at $7^{\circ} \mathrm{C}$. In both cultivars, watermelons grafted on Ferro and RS841 rootstocks preserved their postharvest quality better than non-grafted fruits and other rootstocks (Özdemir et al., 2016, 2018).

The aim of this study was to carry out the effects of low temperature storage on chilling injury and other quality criteria's of watermelon fruit from Crimson Tide and Crisby grafted onto Ferro, RS841, Argentario, and Macis rootstocks during storage at $0^{\circ} \mathrm{C}$ and $90 \pm 5 \%$ relative humidity for 21 days and shelf life at $21 \pm 0.5^{\circ} \mathrm{C}$ and $70 \pm 5 \%$ relative humidity for 7 days compared to fruit from ungrafted Crimson Tide and Crisby.

\section{Materials and Methods}

\subsection{Materials}

The experiment was carried out at the Republic of Turkey Ministry of Agriculture and Forestry Alata Horticultural Research Institute, Erdemli, Mersin, Turkey. The watermelon [Citrullus lanatus (Thunb.) Matsum. and Nakai] cultivars Crimson Tide (CT) and Crisby (CR) were grafted onto Ferro and RS841 (Cucurbita maxima x Cucurbita moschata) and Argentario and Macis (Lagenaria siceraria) rootstocks by using slunt-cut grafting method (Lee and Oda, 2003). The grafted plants were supplied by the commercial seedling company of Grow Fide (Antalya, Turkey). The non-grafted CT and CR were used as control.

\subsection{Physical, biochemical changes and chilling injury}

Fruits were harvested at full maturity when the $75 \%$ of tendril and stipule on the same node with peduncle were desiccated. After harvest, fruit were stored at $0 \pm 0.5 \mathrm{C}$ and $90 \pm 5 \%$ relative humidity for 21 days in cold store and hold 21 days at $0{ }^{\circ} \mathrm{C}$ and subsequent 7 days at $21 \pm 0.5^{\circ} \mathrm{C}$ and $70 \pm 5 \%$ relative humidity for shelf life.

Changes in weight loss (\%), the incidence of fungal decay (\%), fruit flesh firmness $(\mathrm{N})$, total soluble solids (\%), juice $\mathrm{pH}$, titratable acidity (\%), ripening (1-7), citric and malic acid (\%), lycopene $\left(\mu \mathrm{g} \mathrm{g}^{-1}\right), \beta$-carotene $\left(\mu \mathrm{g} \mathrm{g}^{-1}\right)$, hallow heart $(1-5)$, fructose $(\%)$, glucose $(\%)$, sucrose $(\%)$, total sugar $(\%)$, sensory quality (1-9), flesh colour $\left(L^{*}, C^{*}\right.$ and $\left.\mathrm{h}^{\circ}\right)$ values, chilling injury in rind (external) and flesh (internal) (1-5) were determined during storage and shelf life at a weekly interval.

Weight loss (\%); 30 fruit were numbered and the weight loss was determined in reference to initial weight of fruit with a laboratory balance sensitive until $0.01 \mathrm{~g}$ for grafted and ungrafted fruit of both cultivars. Fruit flesh firmness $(\mathrm{N})$; the heart portion of the fruit was measured with a penetrometer (Now FHR-5 Nippon Optical Works Co. Ltd. Tokyo, Japan) having a drilling head at a conical probe of $12-\mathrm{mm}$ in the force in kilograms and the results were translated into newton (N). Total soluble solid (TSS) content (\%); TSS content was determined on juice obtained from 5 watermelons per replicate with the help of a handheld refractometer (Atago Model ATC-1E Atago Co. Ltd., Tokyo, Japan) at $20^{\circ} \mathrm{C}$ and calculated in percent. juice $\mathrm{pH} ; \mathrm{pH}$ was measured by digital $\mathrm{pH}$-meter (Orion 5-Star model Thermo Fisher Scientific Inc., MA, ABD). Titratable acidity (TA) content (\%); TA content was measured by employing potentiometric method for measuring TA content, $5 \mathrm{ml}$ of fruit juice obtained from 5 watermelons per replicate were completed until 100 $\mathrm{ml}$ and titrated with $0.1 \mathrm{~N} \mathrm{NaOH}$ to $\mathrm{pH} 8.1$ (expressed as g malic acid per $100 \mathrm{~mL}$ juice). The fruits were also scored at each evaluation for chilling injury $(\mathrm{Cl})$ in the rind (external) and flesh (internal) and decay $(1=$ none, $2=<10 \%$ of surface area, $3=$ $11 \%$ to $25 \%, 4=26 \%$ to $50 \%$, and $5=>50 \%$ ) (Risse et al., 1990). Incidence of $\mathrm{Cl}$ and decay were determined after 7,14 or 21 days at $0^{\circ} \mathrm{C}$ and 7 days at $21^{\circ} \mathrm{C}$ following each storage duration. Sensory quality (1-9) of fruit was rated with 1-9 hedonic scale. 1: very bad and 9: the best values show on this scale, hallow heart $(1-5)$ of fruit were rated on hedonic scale of $1=$ none to $5=$ very severe $(50 \%$ "more than hallow heart) and ripening (1-7) of fruit were rated on hedonic scale of $1=$ raw fruit and $3=$ mature to $7=0$ ver-ripe extremely by trained ten panelists. Fruit flesh color was assessed as reflected in the CIELAB $\left(\mathrm{L}^{*} \mathrm{a}^{*} \mathrm{~b}^{*}\right)$ color space using a CR-300 Minolta Chroma Meter (Konica Minolta, Osaka, Japan), calibrated using the manufacturer's standard white plate. Twice reading was made from the flesh of fruit. Flesh color $L^{*}$ value indicates lightness, ranging from 100 (white) to 0 (black). Flesh color Chroma $\left(\mathrm{C}^{*}\right)$ value indicates color saturation, which varies from matt (poor value) to vivid color (top value) and it was computed using the formula $(a 2+b 2) 1 / 2$. Flesh color Hue angle $\left(h^{\circ}\right)$ value defines an angle from a color wheel with redpurple $\left(0^{\circ}\right)$, yellow $\left(90^{\circ}\right)$, bluish green $\left(180^{\circ}\right)$, and blue $\left(270^{\circ}\right)$, and it was determined by $h^{\circ}=\tan -1$ (b/a), (McGuire, 1992).

Sugars and organic acids analysis were performed in homogenized frozen watermelon samples. For this process, the samples were slipped through Whatman No. 4 filter paper under vacuum and $20 \mu \mathrm{L}$ of watermelon sample was syringed immediately into the HPLC (LC-10A Series, Shimadzu Co., Kyoto, Japan) equipment. The HPLC analysis of sugars were performed on 
equipment consisting of a refractive index detector and Nucleosil Carbohydrate column $(250 \mathrm{~mm} \times 4 \mathrm{~mm}$, Macherey-Nagel, Düren, Germany) with $2 \mathrm{~mL} \min ^{-1}$ flow rate at $25^{\circ} \mathrm{C}$, all of the sugars analysis was at $210 \mathrm{~nm}$. The HPLC analysis were determined on equipment including of a photodiode array detector of organic acids and a TransgenomicTM ICSep ION 300 column (300 mm $\times 7.8 \mathrm{~mm}$, Transgenomic San Jose, CA, USA) with $0.4 \mathrm{~mL} \mathrm{~min}^{-1}$ flow rate at $65^{\circ} \mathrm{C}$, all of the organic acids analysis was at $210 \mathrm{~nm}$ (Chisholm and Picha, 1986). For sugar analysis of the mobile phase was comprised of acetonitrile and water at

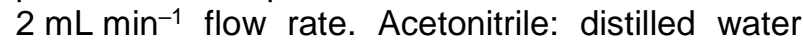
$(80: 20, v / v)$ mixture was used as a mobile phase. For organic acids analysis of the mobile phase was consisted $0.0085 \mathrm{~N} \mathrm{H}_{2} \mathrm{SO}_{4}$ at $0.4 \mathrm{~mL} \mathrm{~min}^{-1}$ flow

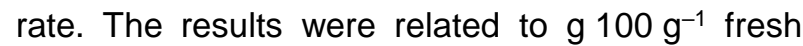
weight.

Analysis of carotenoids was performed in homogenized frozen watermelon samples. For this process, $t$ the homogenization of the frozen watermelon samples were done using a $10 \mathrm{~mm}$ shaft and a low speed of Ultra-Turrax homogenizer. The purees $(3 \mathrm{~g})$ were taken into the centrifuge tube and obtained with HPLC-grade solvents of $5 \mathrm{~mL}$ of ethanol, $5 \mathrm{~mL}$ of acetone and $10 \mathrm{~mL}$ of hexane including $0.05 \%$ butylated hydroxytoluene (Merck $\mathrm{KGaA}$ ) and $20 \mu \mathrm{L}$ of the sample, which was the top hexane layer was filtrated with a $0.45-\mu \mathrm{m}$ Millex-HV filter (Millipore), was syringed instantly into Shimadzu HPLC equipment (LC-10A Series, Shimadzu Co., Kyoto, Japan). The HPLC analysis of carotenoids were performed on equipment consisting of a photodiode array detector and a YMC carotenoid C30 column $(250 \mathrm{~mm} \times 4.6 \mathrm{~mm}$, YMC Europe GMBH) with $1.5 \mathrm{~mL} \mathrm{~min}^{-1}$ flow rate at $30^{\circ} \mathrm{C}$, the lycopene analysis was at $503 \mathrm{~nm}$ and the $\beta$-carotene analysis was at $452 \mathrm{~nm}$ (Perkins-Veazie and Collins 2006). For carotenoids analysis of the mobile phase was comprised of methyl tertiary butyl ether, methanol and deionized distilled water $(15: 81: 41$, solvent $A)$, methyl tertiary butyl ether and methanol (90:10, solvent B) (Liu et al., 2009). The results were defined as $\mu \mathrm{g} \mathrm{g}^{-1}$ fresh weight.

\subsection{Statistical analysis}

The study was carried out during a 2-year period and data are expressed as the mean of 2 experimental years. The data were analysed a completely randomized block design by ANOVA using SAS software of SAS Institute, Cary, N.C. (SAS, 2019). The data were obtained from three replicates per scion/rootstock combination. Each replicates contained 5 fruit. The mean separation at $P<0.05$ level was made with Fisher's Least Significance Test.

\section{Results and Discussion}

Weight loss in grafted and control fruit were very low $(<1 \%)$ during storage for both cultivars. In CT cultivar, fruit on RS841 rootstock resulted in higher weight loss than those on other rootstocks and control fruit at the end of the storage time and control fruit resulted in higher weight loss than those on other rootstocks at the end of the storage time and shelf life. In CR cultivar, fruit on RS841 rootstock and control fruit resulted in higher weight loss than those on other rootstocks at the end of the storage time and fruit on RS841 rootstock and control fruit resulted in higher weight loss than those on other rootstocks during the storage time and shelf life periods (Figure 1, 2, 3 and 4). Consistent with our results, Perkins-Veazie and Collins (2006) and Özdemir et al. $(2016,2018)$ reported the $<1 \%$ of weight loss in watermelon fruit during storage or shelf life. However, Araújo Neto et al. (2000) determined higher weight loss $(3.8 \%)$ than our results. Suárez-Hernández et al. (2016) reported that some rootstocks caused to reduce in weight loss during storage periods.

Fungal decay was not observed during storage for both cultivars but, except during the shelf life. The decayed areas covered $<10 \%$ of rind surface of fruit. The graft combinations did not differ in the incidence of fungal during shelf life for both cultivars (Figure 5 and 6). Fungal decay that occurred during shelf life after storage at $0^{\circ} \mathrm{C}$ might be due to

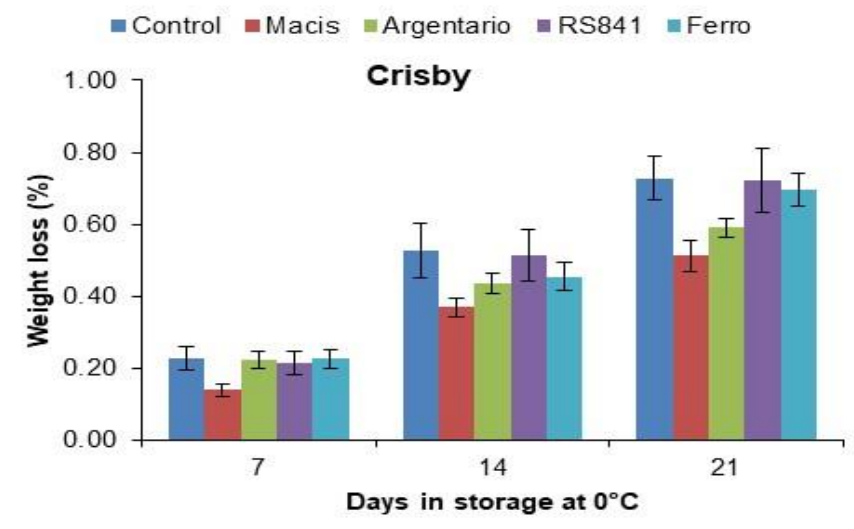

Figure 1. The effects of rootstocks on weight loss of Crisby watermelon fruits during storage 


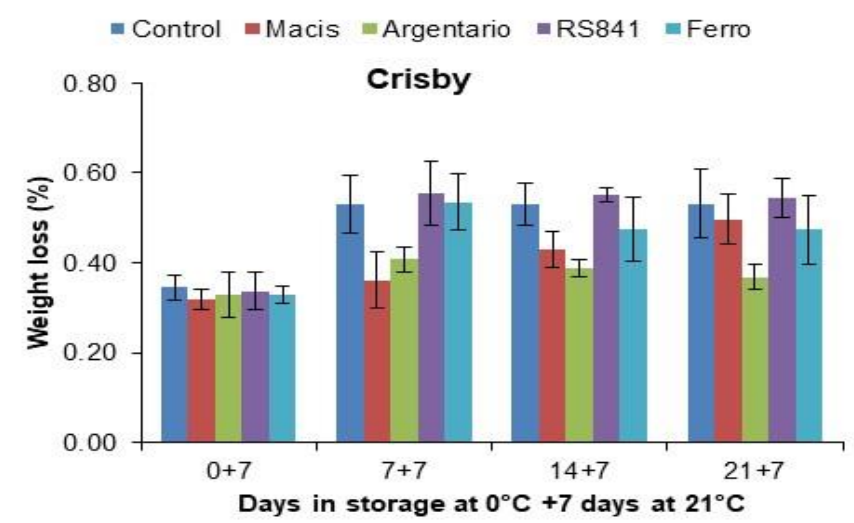

Figure 2. The effects of rootstocks on weight loss of Crisby watermelon fruits during shelf life

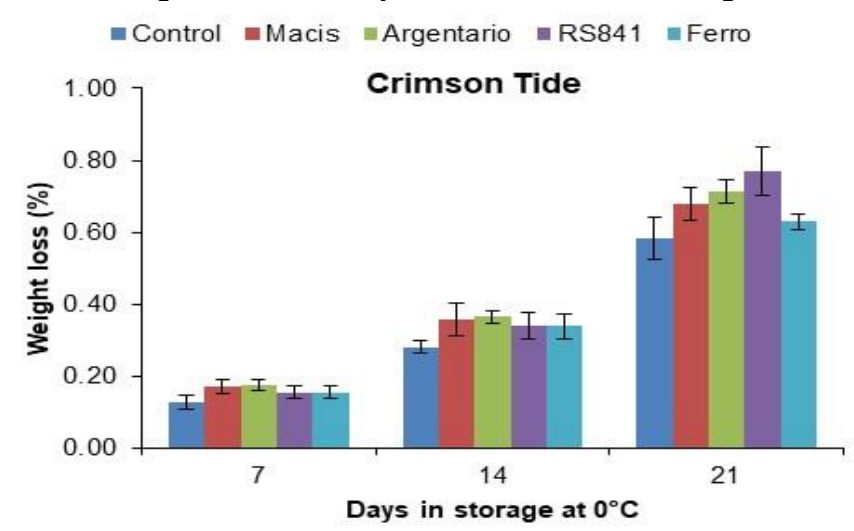

Figure 3. The effects of rootstocks on weight loss of Crimson Tide watermelon fruits during storage

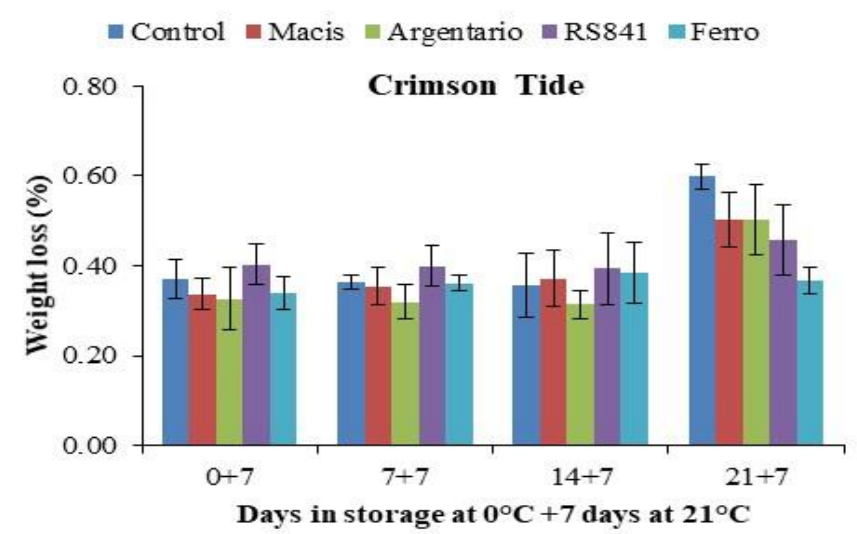

Figure 4. The effects of rootstocks on weight loss of Crimson Tide watermelon fruits during shelf life

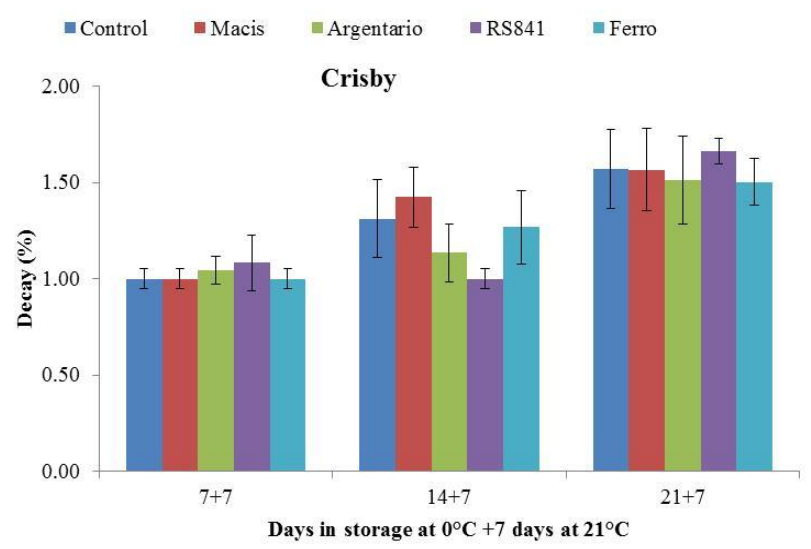

Figure 5. The effects of rootstocks on fungal decay of Crisby watermelon fruits during shelf life 


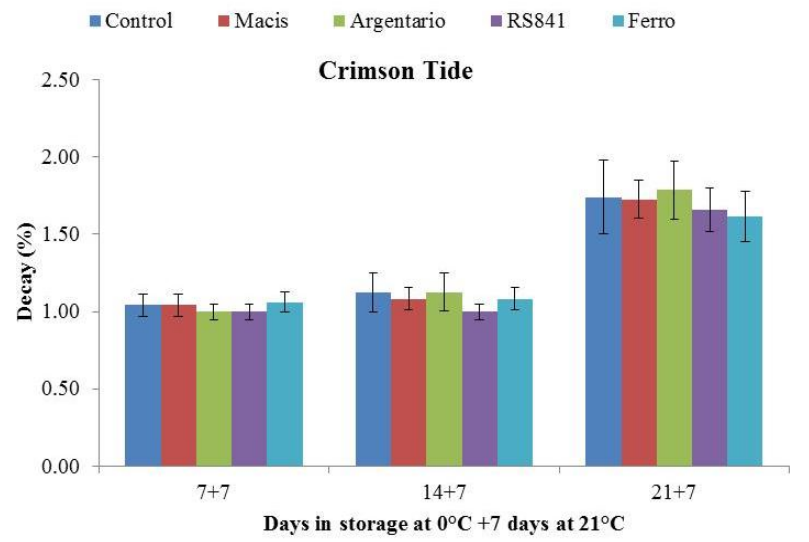

Figure 6. The effects of rootstocks on fungal decay of Crimson Tide watermelon fruits during shelf life

increased susceptibility of fruit to decay due to $\mathrm{Cl}$. Risse et al. (1990) reported that most of the decay was observed on the sites of $\mathrm{Cl}$ at 1 and $7^{\circ} \mathrm{C}$ and in small watermelon cultivars, most decay was observed from the stem end at $13^{\circ} \mathrm{C}$ and $21^{\circ} \mathrm{C}$. Similar findings were reported in watermelon fruit by Özdemir et al. (2016, 2018).

Flesh firmness decreased during storage and shelf life for both cultivars. Fruit flesh firmness of watermelons grafted on Ferro and RS841 rootstocks were higher than others in CT and CR cultivars during the storage and shelf life (Table 1 and 2). Consistent with our results, Özdemir et al. (2016, 2018) reported the grafted fruit had firmer comparing to control fruit in watermelons during storage or shelf life.

Suárez-Hernández et al. (2016) reported that the some rootstocks retained firmness better than control fruit during storage. It was reported that at harvest, the fruit flesh firmness of grafted watermelon was higher than control fruits (Soteriou and Kyriacou, 2015; Karaağaç et al., 2018). Watermelon fruit flesh firmness did not change or reduced during storage during 4 weeks of storage at $5,10,15$ or $20^{\circ} \mathrm{C}$ depending on storage temperature and cultivars (Risse et al., 1990). Depending on cultivar, seasonal variation and harvest maturity, postharvest decline in flesh firmness may compromise fruit quality within 14 days from harvest (Kyriacou and Soteriou, 2015). Özdemir et al. (2016) reported that depending on the rootstock and the scion vary the effects of rootstocks on fruit flesh firmness.

TSS content remained above $10 \%$ in fruit of both cultivars throughout storage period (Table 1 and 2), rendering fruit acceptable for perceived sweetness as reported by Kyriacou and Soteriou (2015). In CR cultivar, fruit grafted on Ferro and Argentario rootstocks had higher TSS content after storage period for 21 days at $0{ }^{\circ} \mathrm{C}$, compared to other graft combinations and control. Effect of rootstocks on TSS content was not significant during shelf life (Table 1). In case of CT cultivar, fruit grafted on Ferro and RS-841 rootstocks had higher TSS content during storage, compared to other graft combinations and control. Fruit grafted on RS-841 rootstock had higher TSS content during shelf life, compared to other graft combinations and control (Table 2). Although, some previous studies showed that, grafting on the bottle gourd rootstocks of watermelons raised TSS contents compared to the control fruit (Suárez-Hernández et al., 2016) and grafted watermelons had lower TSS content compared to control (Kyriacou and Soteriou, 2015). In other studies, our reports are consistent with the previous studies, indicating effects of rootstocks on TSS content, cultivar depending Özdemir et al. (2016, 2018).

Juice $\mathrm{pH}$ value slightly decreased during the storage and shelf life (Table 1 and 2). In CR cultivar, effect of rootstocks on $\mathrm{pH}$ value was not significant during storage and control fruit had higher $\mathrm{pH}$ compared to grafted fruit during shelf life (Table 1). In CT cultivar, fruit grafted on Ferro and RS-841 rootstocks had lower $\mathrm{pH}$ compared to other grafted fruit and control during storage and effect of rootstocks on $\mathrm{pH}$ value was not significant during shelf life (Table 2). Our reports are consistent with the previous studies (Özdemir et al., 2016, 2018).

TA content slightly increased in parallel with changes in juice $\mathrm{pH}$ during storage and shelf life for both cultivars during the storage and shelf life (Table 1 and 2). In CR and CT cultivar, fruit on Ferro and $\mathrm{RS} 841$ rootstocks resulted in higher TA than those on other rootstocks and control fruit after 21 days of storage and shelf life (Table 1 and 2). Higher TA due to grafting was reported in watermelon fruit (Proietti et al., 2008; Çandır et al., 2013, Özdemir et al., 2016, 2018).

It was found a slight increase during storage and shelf life for both cultivars in ripening (1-7) ratings (Table 1 and 2), indicating fruit became overripe toward the end of storage. Similar findings were reported by Risse et al. (1990) for several watermelon cultivars during 4 weeks of storage at $5,10,15$ or $20^{\circ} \mathrm{C}$. In CR cultivar, fruit grafted on RS841 rootstock had lower ripening scores than those from other rootstocks and control fruit after 21 days of storage and effect of rootstocks on ripening ratings were not significant during shelf life (Table 1). In CT cultivars all grafted fruit had lower ripening scores, compared to control fruit after 21 days of 
Table 1. The effects of rootstocks on fruit flesh firmness (N), TSS (\%), juice pH, TA (\%) and ripening (1-7) of Crisby (CR) watermelon fruits during storage at $0^{\circ} \mathrm{C}$ and following 7 days at $21^{\circ} \mathrm{C}$

\begin{tabular}{|c|c|c|c|c|c|c|c|c|c|c|c|}
\hline \multirow{2}{*}{ Parameters } & \multirow{2}{*}{ Scion/ rootstock } & \multicolumn{4}{|c|}{ Days in storage at $0^{\circ} \mathrm{C}$} & \multirow{2}{*}{ Mean } & \multicolumn{4}{|c|}{ Days in shelf life at $21^{\circ} \mathrm{C}$} & \multirow{2}{*}{ Mean } \\
\hline & & 0 & 7 & 14 & 21 & & $0+7$ & $7+7$ & $14+7$ & $21+7$ & \\
\hline \multirow{5}{*}{$\begin{array}{l}\text { Firmness } \\
(\mathrm{N})\end{array}$} & $\mathrm{CR}($ & $7.84 \mathrm{~b}$ & $6.81 \mathrm{c}$ & $5.67 c$ & $5.49 \mathrm{c}$ & $6.45 \mathrm{~d}$ & $6.16 \mathrm{~d}$ & $4.89 \mathrm{c}$ & $4.14 \mathrm{c}$ & $3.87 \mathrm{c}$ & $4.77 \mathrm{c}$ \\
\hline & $\mathrm{CR} / \mathrm{N}$ & $7.57 \mathrm{c}$ & $7.40 \mathrm{bc}$ & $6.72 \mathrm{~b}$ & $6.18 \mathrm{~b}$ & $6.97 \mathrm{c}$ & $6.31 \mathrm{~cd}$ & $6.69 \mathrm{~b}$ & $5.59 \mathrm{~b}$ & $5.23 \mathrm{~b}$ & \\
\hline & $\mathrm{CR} / \mathrm{Arg}$ & $8.13 \mathrm{a}$ & $7.79 a b$ & $6.74 \mathrm{~b}$ & $6.37 \mathrm{~b}$ & $7.26 \mathrm{bc}$ & $6.86 \mathrm{bc}$ & $6.75 \mathrm{~b}$ & $6.28 \mathrm{a}$ & $5.43 \mathrm{~b}$ & \\
\hline & $\mathrm{CR} / \mathrm{P}$ & $26 \mathrm{a}$ & $8.40 \mathrm{a}$ & $7.57 \mathrm{a}$ & $7.12 \mathrm{a}$ & $7.84 \mathrm{a}$ & $7.65 \mathrm{a}$ & $7.50 \mathrm{a}$ & $6.86 \mathrm{a}$ & $6.62 \mathrm{a}$ & \\
\hline & & $.24 \mathrm{a}$ & $7.91 \mathrm{ab}$ & $6.96 \mathrm{~b}$ & $7.01 \mathrm{a}$ & $7.53 \mathrm{~b}$ & $7.47 \mathrm{ab}$ & $6.80 \mathrm{~b}$ & $6.86 \mathrm{a}$ & $6.55 \mathrm{a}$ & \\
\hline \multirow{5}{*}{$\begin{array}{l}\text { TSS } \\
(\%)\end{array}$} & & $0.30 \mathrm{a}$ & $10.40 \mathrm{~b}$ & $11.20 \mathrm{a}$ & $10.60 \mathrm{~b}$ & & $10.60 \mathrm{bc}$ & $10.60 \mathrm{a}$ & $11.10 \mathrm{a}$ & $10.80 \mathrm{a}$ & $30 \mathrm{a}$ \\
\hline & & $0.20 \mathrm{a}$ & & $11.10 \mathrm{a}$ & $10.40 \mathrm{~b}$ & & $10.20 \mathrm{c}$ & $10.50 \mathrm{a}$ & $10.90 \mathrm{a}$ & 10.70 & \\
\hline & & $0.60 \mathrm{a}$ & & $11.10 \mathrm{a}$ & $11.20 \mathrm{a}$ & & $10.80 \mathrm{~b}$ & $10.20 \mathrm{a}$ & 11.00 & 10.50 & \\
\hline & & $50 \mathrm{a}$ & 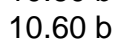 & 1 & . & & $10.90 \mathrm{ab}$ & $10.50 \mathrm{a}$ & & 10.90 & \\
\hline & & $00 \mathrm{a}$ & & $20 a$ & $11.30 \mathrm{a}$ & & $11.40 \mathrm{a}$ & $10.40 \mathrm{a}$ & & $10.80 \mathrm{c}$ & 0 a \\
\hline \multirow{5}{*}{$\begin{array}{l}\text { Juice } \\
\mathrm{pH}\end{array}$} & & $5 a$ & $5.69 \mathrm{bc}$ & $5.69 \mathrm{a}$ & $5.57 \mathrm{a}$ & & $80 \mathrm{a}$ & $5.65 \mathrm{a}$ & $5.66 \mathrm{a}$ & $5.74 \mathrm{a}$ & \\
\hline & & $65 a$ & 5.64 c & $5.64 \mathrm{a}$ & $5.57 \mathrm{a}$ & $5.62 \mathrm{a}$ & $5.63 \mathrm{~b}$ & $5.53 \mathrm{~b}$ & $5.51 \mathrm{~b}$ & $5.63 \mathrm{~b}$ & $b c$ \\
\hline & $\mathrm{CF}$ & $67 \mathrm{a}$ & $5.74 a b$ & $5.61 \mathrm{a}$ & $5.67 \mathrm{a}$ & & $5.64 \mathrm{~b}$ & $5.59 a b$ & $5.58 a b$ & $5.68 \mathrm{ab}$ & \\
\hline & & $.58 \mathrm{a}$ & $5.69 \mathrm{bc}$ & $5.60 \mathrm{a}$ & $5.58 \mathrm{a}$ & & $5.62 \mathrm{~b}$ & $5.51 \mathrm{~b}$ & $5.49 \mathrm{~b}$ & $5.55 \mathrm{~b}$ & \\
\hline & & $54 a$ & $5.82 \mathrm{a}$ & $5.58 \mathrm{a}$ & $5.66 \mathrm{a}$ & & $5.65 b$ & $5.54 \mathrm{~b}$ & $5.54 \mathrm{~b}$ & $5.63 c$ & $9 \mathrm{bc}$ \\
\hline \multirow{5}{*}{$\begin{array}{l}\text { TA } \\
(\%)\end{array}$} & & $5 a$ & $0.17 \mathrm{a}$ & $0.17 \mathrm{bc}$ & $0.16 \mathrm{a}$ & & $16 \mathrm{~b}$ & $0.18 \mathrm{a}$ & $0.16 \mathrm{~b}$ & $0.16 \mathrm{~b}$ & $b$ \\
\hline & & $15 a$ & $0.15 \mathrm{a}$ & $0.16 \mathrm{c}$ & $0.14 \mathrm{a}$ & & $0.16 \mathrm{~b}$ & $0.16 \mathrm{a}$ & $0.16 \mathrm{~b}$ & $0.17 a b$ & $6 \mathrm{~b}$ \\
\hline & $\mathrm{CF}$ & $0.14 \mathrm{a}$ & $0.16 \mathrm{a}$ & $0.16 \mathrm{c}$ & $0.15 \mathrm{a}$ & & $0.16 \mathrm{~b}$ & $0.16 \mathrm{a}$ & $0.16 \mathrm{~b}$ & $0.16 \mathrm{~b}$ & $6 \mathrm{~b}$ \\
\hline & & $0.15 \mathrm{a}$ & $0.17 \mathrm{a}$ & $0.18 \mathrm{a}$ & $0.16 \mathrm{a}$ & & $0.18 \mathrm{a}$ & $0.18 \mathrm{a}$ & $0.18 a b$ & $0.19 a$ & \\
\hline & & $16 a$ & $0.16 \mathrm{a}$ & $0.18 \mathrm{a}$ & $0.16 \mathrm{a}$ & & $0.17 \mathrm{ab}$ & $0.18 \mathrm{a}$ & $0.19 a$ & $0.18 \mathrm{a}$ & \\
\hline \multirow{5}{*}{$\begin{array}{l}\text { Ripening } \\
(1-7)\end{array}$} & & $70 \mathrm{a}$ & $3.60 \mathrm{a}$ & $3.40 \mathrm{a}$ & $3.70 \mathrm{a}$ & & $4.00 \mathrm{a}$ & $4.50 \mathrm{a}$ & $3.60 \mathrm{a}$ & $3.70 \mathrm{a}$ & \\
\hline & & $3.20 \mathrm{~b}$ & $3.50 \mathrm{a}$ & $3.40 \mathrm{a}$ & $3.70 \mathrm{a}$ & $3.40 a b$ & $3.80 \mathrm{a}$ & $3.40 \mathrm{c}$ & $3.70 \mathrm{a}$ & $4.10 \mathrm{a}$ & $3.80 \mathrm{a}$ \\
\hline & CR/Argentario & $3.60 \mathrm{a}$ & $3.40 \mathrm{a}$ & $3.50 \mathrm{a}$ & $3.90 \mathrm{a}$ & $3.60 \mathrm{a}$ & $3.80 \mathrm{a}$ & $3.80 \mathrm{~b}$ & $3.80 \mathrm{a}$ & $3.90 \mathrm{a}$ & $3.80 \mathrm{a}$ \\
\hline & & $3.10 \mathrm{~b}$ & $3.20 \mathrm{a}$ & $3.30 \mathrm{a}$ & $3.50 \mathrm{a}$ & $3.20 \mathrm{~b}$ & $3.50 \mathrm{a}$ & $3.40 \mathrm{c}$ & $3.50 \mathrm{a}$ & $3.70 \mathrm{a}$ & $3.50 \mathrm{a}$ \\
\hline & CR/Ferro & $3.30 a b$ & $3.30 \mathrm{a}$ & $3.70 \mathrm{a}$ & $3.50 \mathrm{a}$ & $3.40 \mathrm{a}$ & $3.60 \mathrm{a}$ & $3.50 \mathrm{c}$ & $3.60 \mathrm{a}$ & $3.60 \mathrm{a}$ & $3.60 \mathrm{a}$ \\
\hline
\end{tabular}

$\mathrm{x}_{\text {Mean separation was performed by Fisher's LSD test. Means }(n=3) \text { followed by same letters within a column are not significantly different }}$ at $\mathrm{P}<0.05$

Table 2. The effects of rootstocks on fruit flesh firmness (N), TSS (\%), juice pH, TA (\%) and ripening (1-7) of Crimson Tide (CT) watermelon fruits during storage at $0^{\circ} \mathrm{C}$ and following 7 days at $21^{\circ} \mathrm{C}$

\begin{tabular}{|c|c|c|c|c|c|c|c|c|c|c|c|}
\hline \multirow{2}{*}{ Parameters } & \multirow{2}{*}{ Scion/ rootstock } & \multicolumn{4}{|c|}{ Days in storage at $0^{\circ} \mathrm{C}$} & \multirow{2}{*}{ Mean } & \multicolumn{4}{|c|}{ Days in shelf life at $21^{\circ} \mathrm{C}$} & \multirow{2}{*}{ Mean } \\
\hline & & 0 & 7 & 14 & 21 & & $0+7$ & $7+7$ & $14+7$ & $21+7$ & \\
\hline \multirow{5}{*}{$\begin{array}{l}\text { Firmness } \\
\text { (N) }\end{array}$} & CT(Control) & $7.37 \mathrm{c}$ & $6.23 \mathrm{~b}$ & $6.28 c$ & $5.86 \mathrm{~b}$ & $6.43 c$ & $5.74 \mathrm{c}$ & $6.16 \mathrm{c}$ & $5.18 \mathrm{c}$ & $4.85 \mathrm{c}$ & $5.48 \mathrm{e}$ \\
\hline & CT/Macis & $7.75 \mathrm{bc}$ & $6.95 \mathrm{ab}$ & $6.08 \mathrm{c}$ & $5.89 \mathrm{~b}$ & $6.67 \mathrm{bc}$ & $6.06 \mathrm{c}$ & $6.06 \mathrm{c}$ & $5.64 \mathrm{bc}$ & $5.69 \mathrm{~b}$ & $5.86 \mathrm{~d}$ \\
\hline & CT/Argentario & $7.96 \mathrm{~b}$ & $7.08 \mathrm{a}$ & $6.59 \mathrm{bc}$ & $5.91 \mathrm{~b}$ & $6.88 \mathrm{~b}$ & $7.03 \mathrm{~b}$ & $6.74 \mathrm{~b}$ & $5.85 b$ & $5.86 \mathrm{~b}$ & $6.37 c$ \\
\hline & CT/RS841 & $8.31 \mathrm{ab}$ & $7.60 \mathrm{a}$ & $7.13 a b$ & $6.92 \mathrm{a}$ & $7.49 \mathrm{a}$ & $7.16 \mathrm{~b}$ & $6.78 \mathrm{~b}$ & $6.59 \mathrm{a}$ & $7.24 \mathrm{a}$ & $6.94 \mathrm{~b}$ \\
\hline & CT/Ferro & $8.55 \mathrm{a}$ & $7.68 \mathrm{a}$ & $7.53 \mathrm{a}$ & $7.06 \mathrm{a}$ & $7.70 \mathrm{a}$ & $7.99 \mathrm{a}$ & $7.52 \mathrm{a}$ & $7.04 \mathrm{a}$ & $7.28 \mathrm{a}$ & $7.46 \mathrm{a}$ \\
\hline \multirow{5}{*}{$\begin{array}{l}\text { TSS } \\
(\%)\end{array}$} & CT(Control) & $11.10 \mathrm{a}$ & $11.10 \mathrm{a}$ & $11.20 \mathrm{a}$ & $11.10 \mathrm{ab}$ & $11.10 \mathrm{a}$ & $10.90 \mathrm{a}$ & $10.70 \mathrm{~b}$ & $10.60 \mathrm{a}$ & $10.90 \mathrm{~b}$ & $10.80 \mathrm{~b}$ \\
\hline & CT/Macis & $10.60 \mathrm{a}$ & $10.90 \mathrm{a}$ & $10.70 \mathrm{a}$ & $10.30 \mathrm{c}$ & $10.60 \mathrm{c}$ & $10.60 \mathrm{a}$ & $10.70 \mathrm{~b}$ & $10.40 \mathrm{a}$ & $10.80 \mathrm{k}$ & $10.60 \mathrm{~b}$ \\
\hline & CT/Argentario & $10.80 \mathrm{a}$ & $11.20 \mathrm{a}$ & $10.50 \mathrm{a}$ & $10.60 \mathrm{bc}$ & $10.80 \mathrm{bc}$ & $10.10 \mathrm{~b}$ & $10.80 a b$ & $10.60 \mathrm{a}$ & $11.00 \mathrm{k}$ & $10.60 \mathrm{~b}$ \\
\hline & CT/RS841 & $10.90 \mathrm{a}$ & $11.50 \mathrm{a}$ & & $10.80 a b$ & $11.00 \mathrm{ab}$ & $10.90 \mathrm{a}$ & $11.30 \mathrm{a}$ & $11.30 \mathrm{a}$ & 11.60 & $11.30 \mathrm{a}$ \\
\hline & CT/Ferro & $10.60 \mathrm{a}$ & $11.20 \mathrm{a}$ & $11.10 \mathrm{a}$ & $11.20 \mathrm{a}$ & $11.00 \mathrm{ab}$ & $10.80 \mathrm{a}$ & $10.40 \mathrm{~b}$ & $10.90 \mathrm{a}$ & $10.70 \mathrm{~b}$ & $10.70 \mathrm{~b}$ \\
\hline \multirow{5}{*}{$\begin{array}{l}\text { Juice } \\
\mathrm{pH}\end{array}$} & $\mathrm{CT}$ (Control) & $5.67 \mathrm{a}$ & $5.63 \mathrm{a}$ & $5.71 \mathrm{a}$ & $5.72 \mathrm{ab}$ & $5.68 \mathrm{a}$ & $5.69 \mathrm{a}$ & $5.56 \mathrm{a}$ & $5.53 \mathrm{a}$ & $5.74 \mathrm{a}$ & $5.63 \mathrm{a}$ \\
\hline & CT/Macis & $5.66 \mathrm{a}$ & $5.64 \mathrm{a}$ & $5.64 a b$ & $5.78 \mathrm{a}$ & $5.68 \mathrm{a}$ & $5.60 \mathrm{a}$ & $5.53 \mathrm{a}$ & $5.52 \mathrm{a}$ & $5.73 \mathrm{a}$ & $5.59 \mathrm{a}$ \\
\hline & CT/Argentario & $5.69 \mathrm{a}$ & $5.62 \mathrm{a}$ & $5.57 \mathrm{bc}$ & $5.76 \mathrm{a}$ & $5.66 \mathrm{a}$ & $5.62 \mathrm{a}$ & $5.43 \mathrm{bc}$ & $5.58 \mathrm{a}$ & $5.70 \mathrm{a}$ & $5.58 \mathrm{a}$ \\
\hline & $\mathrm{CT} / \mathrm{R}$ & $5.66 \mathrm{a}$ & $5.53 \mathrm{ab}$ & $5.48 c$ & $5.55 \mathrm{~b}$ & $5.55 \mathrm{~b}$ & $5.59 \mathrm{a}$ & $5.50 a b$ & $5.46 \mathrm{a}$ & $5.62 \mathrm{a}$ & $5.54 \mathrm{a}$ \\
\hline & CT/Ferro & $5.56 \mathrm{a}$ & $5.47 \mathrm{~b}$ & $5.50 \mathrm{c}$ & $5.57 \mathrm{~b}$ & $5.53 \mathrm{~b}$ & $5.61 \mathrm{a}$ & $5.42 \mathrm{c}$ & $5.50 \mathrm{a}$ & $5.63 \mathrm{a}$ & $5.54 \mathrm{a}$ \\
\hline \multirow{5}{*}{$\begin{array}{l}\text { TA } \\
(\%)\end{array}$} & CT(Control) & $0.17 \mathrm{ab}$ & $0.19 \mathrm{~b}$ & $0.16 \mathrm{~b}$ & $0.17 \mathrm{a}$ & $0.17 \mathrm{~b}$ & $0.17 \mathrm{a}$ & $0.18 \mathrm{a}$ & $0.18 \mathrm{~b}$ & $0.17 \mathrm{~b}$ & $0.18 \mathrm{~b}$ \\
\hline & CT/Macis & $0.16 \mathrm{~b}$ & $0.15 c$ & $0.14 \mathrm{c}$ & $0.14 \mathrm{~b}$ & $0.15 \mathrm{c}$ & $0.17 \mathrm{a}$ & $0.16 \mathrm{a}$ & $0.16 \mathrm{c}$ & $0.18 a b$ & $0.17 \mathrm{~b}$ \\
\hline & CT/Argentario & $0.16 \mathrm{~b}$ & $0.17 \mathrm{bc}$ & $0.15 b c$ & $0.15 a b$ & $0.16 \mathrm{c}$ & $0.16 \mathrm{a}$ & $0.18 \mathrm{a}$ & $0.16 \mathrm{c}$ & $0.17 \mathrm{~b}$ & $0.17 b$ \\
\hline & CT/RS841 & $0.17 a b$ & $0.19 b$ & $0.18 \mathrm{a}$ & $0.17 \mathrm{a}$ & $0.18 a b$ & $0.17 \mathrm{a}$ & $0.18 \mathrm{a}$ & $0.20 \mathrm{a}$ & $0.21 \mathrm{a}$ & $0.19 \mathrm{a}$ \\
\hline & CT/Ferro & $0.18 \mathrm{a}$ & $0.21 \mathrm{a}$ & $0.18 \mathrm{a}$ & $0.17 \mathrm{a}$ & $0.19 \mathrm{a}$ & $0.18 \mathrm{a}$ & $0.18 \mathrm{a}$ & $0.19 a b$ & $0.21 \mathrm{a}$ & $0.18 \mathrm{a}$ \\
\hline \multirow{5}{*}{$\begin{array}{l}\text { Ripening } \\
(1-7)\end{array}$} & CT(Control) & $3.30 \mathrm{a}$ & $3.80 \mathrm{a}$ & $4.40 \mathrm{a}$ & $4.10 \mathrm{a}$ & $3.90 \mathrm{a}$ & $4.60 \mathrm{a}$ & $4.20 \mathrm{a}$ & $4.70 \mathrm{a}$ & $5.70 \mathrm{a}$ & $4.80 \mathrm{a}$ \\
\hline & CT/Macis & $3.30 \mathrm{a}$ & $3.70 \mathrm{a}$ & $4.10 \mathrm{a}$ & $4.20 \mathrm{a}$ & $3.80 \mathrm{a}$ & $4.40 a b$ & $4.10 \mathrm{a}$ & $4.00 \mathrm{a}$ & $5.00 \mathrm{~b}$ & $4.40 \mathrm{~b}$ \\
\hline & CT/Argentario & $3.20 \mathrm{a}$ & $3.30 \mathrm{a}$ & $3.70 \mathrm{~b}$ & $4.10 \mathrm{a}$ & $3.50 \mathrm{~b}$ & $3.30 \mathrm{~b}$ & $3.60 \mathrm{~b}$ & $4.20 \mathrm{a}$ & $5.10 \mathrm{~b}$ & $4.00 \mathrm{c}$ \\
\hline & CT/RS841 & $3.10 \mathrm{a}$ & $3.40 \mathrm{a}$ & $3.60 \mathrm{bc}$ & $3.90 \mathrm{a}$ & $3.50 \mathrm{~b}$ & $3.70 \mathrm{~b}$ & $3.70 \mathrm{~b}$ & $4.00 \mathrm{a}$ & $4.80 \mathrm{bc}$ & $4.10 \mathrm{bc}$ \\
\hline & CT/Ferro & $3.10 \mathrm{a}$ & $3.20 \mathrm{a}$ & $3.30 \mathrm{c}$ & $3.60 \mathrm{a}$ & $3.30 \mathrm{c}$ & $3.30 \mathrm{~b}$ & $3.30 \mathrm{c}$ & $3.80 \mathrm{a}$ & $4.40 \mathrm{c}$ & $3.70 \mathrm{~d}$ \\
\hline
\end{tabular}

${ }^{x}$ Mean separation was performed by Fisher's LSD test. Means $(n=3)$ followed by same letters within a column are not significantly different at $\mathrm{P}<0.05$. 
storage and shelf life. Moreover, in CT cultivar, fruit grafted on Ferro rootstock had lowest ripening scores in all grafted and control fruit after 21 days of storage and shelf life (Table 2). Ripening could be retarded by grafting in watermelon fruit at harvest (Özdemir et al., 2016, 2018). Soteriou et al. (2014) found that as grafting retarded the ripening process, optimum harvest maturity in non-grafted plant was reached 35-40 days post-anthesis (dpa) compared with 40-45 dpa in grafted plants. Similarly, Özdemir et al. (2016) reported that fruit grafted on RS841 and Ferro rootstocks for CR cultivar and fruit grafted on RS841, Argentario and Ferro rootstocks for CT cultivar had the lowest ripening ratings after shelf life period following storage.

In CT cultivar, the citric acid amount ranged between $0.06-0.09 \%$ during storage and $0.06 \%$ to $0.10 \%$ for CT cultivar and the malic acid content ranged from $0.19 \%$ to $0.25 \%$ for CR cultivar and $0.21 \%$ to $0.32 \%$ for CT cultivar after 21 days of storage and shelf life (Table 3 and 4 ). In CR cultivar, fruit grafted on Ferro rootstock had higher citric and malic acid content than those from other rootstocks and control fruit after 21 days of storage and fruit grafted on RS841 and Ferro rootstocks had higher citric and malic acid content than those from other rootstocks and control fruit during shelf life (Table 3). In CT cultivar, fruit grafted on RS841 and Ferro rootstocks and control fruit had higher citric acid content than those from other rootstocks after 21 days of storage and fruit grafted on RS841 rootstock had higher citric acid content than those from other rootstocks and control fruit during shelf life (Table 4). Malic acid was the predominant organic acid for both cultivars. In CT cultivar, fruit grafted on RS841 and Ferro rootstocks had higher malic acid content than those from other rootstocks after 21 days of storage and shelf life (Table 4). In similarly to our findings, it was reported malic acid is the predominant organic acid in watermelon fruit by Özdemir et al. $(2016,2018)$.

Chilling injury $(\mathrm{Cl})$ typically occurs after storage at temperatures $<7^{\circ} \mathrm{C}$ in watermelon fruit (Özdemir et al., 2016, 2018). Symptoms of chilling injury include pitting, decline in flesh color, loss of flavour, off-flavours and increased decay when returned to room temperatures (Suslow, 1997). In our study, Cl symptoms such as brownish water-soaked areas covered $<25 \%$ of rind surface of fruit during the storage and shelf life for all rootstocks for both cultivars. In CR cultivar, external (rind) $\mathrm{Cl}$ was first observed on fruit grafted on RS841 and Macis rootstocks after 14 days of storage and rind $\mathrm{Cl}$ was observed on all grafted and control fruit after 21 days of storage. Rind $\mathrm{Cl}$ was first observed on fruit grafted on Argentario, RS841 and Ferro rootstocks after $7+7$ days of shelf life and rind $\mathrm{Cl}$ was observed on all grafted and control fruit after $14+7$ days of shelf life. However, the effect of rootstocks and control fruit on the incidence of rind $\mathrm{Cl}$ was not significant after 21 days of storage and shelf life in
CR cultivar (Table 3). In CT cultivar, rind $\mathrm{Cl}$ was first observed on all grafted and control fruit (except fruit grafted on Argentario rootstock) after 14 days of storage and rind $\mathrm{Cl}$ was observed on all grafted and control fruit after 21 days of storage. Rind $\mathrm{Cl}$ was first observed on fruit grafted on Macis, RS841 and Ferro rootstocks after $7+7$ days of shelf life and rind $\mathrm{Cl}$ was observed on all grafted and control fruit after $14+7$ days of shelf life (Figure 7). However, effect of rootstocks and control fruit on the incidence of rind $\mathrm{Cl}$ was not significant after 21 days of storage and shelf life in CT cultivar (Table 4).

Internal (flesh) $\mathrm{Cl}$ was first and only observed on control fruit after 21 days of storage in CR cultivar. Flesh $\mathrm{Cl}$ was first observed on fruit grafted on Argentario rootstock and control fruit after $14+7$ days of shelf life in CR cultivar (Figure 8). Fruit grafted on Ferro rootstocks did not exhibit flesh $\mathrm{Cl}$ symptoms during storage and shelf life in $C R$ cultivar. However, effect of rootstocks and control fruit on the incidence of flesh $\mathrm{Cl}$ was not significant after 21 days of storage and shelf life in CR cultivar (Table 3).

All grafted and control fruit did not exhibit flesh $\mathrm{Cl}$ symptoms during storage in $\mathrm{CT}$ cultivar. Flesh $\mathrm{Cl}$ was first observed on fruit grafted on Argentario rootstock and control fruit after $21+7$ days of shelf life in CT cultivar. Fruit grafted on Macis, RS841 and Ferro rootstocks were not observed flesh $\mathrm{Cl}$ symptoms during shelf life in CT cultivar. However, effect of rootstocks and control fruit on the incidence of flesh $\mathrm{Cl}$ was not significant after 21 days of storage and shelf life in CT cultivar (Table 4). In contrast to our findings, it was reported non-grafted CT and CR or CT and CR grafted onto different rootstocks did not exhibit $\mathrm{Cl}$ symptoms by Özdemir et al. $(2016,2018)$. Picha (1986) evaluated three watermelon cultivars for $\mathrm{Cl}$ at different storage temperatures and durations, and reported less external $\mathrm{Cl}$ developed in fruit stored at $7^{\circ} \mathrm{C}$ than at $0^{\circ} \mathrm{C}$ depending on cultivar. In this study, fruit were stored for 12 days at $7^{\circ} \mathrm{C}$ without loss of marketable fruit. Our data showed that susceptibility to $\mathrm{Cl}$ also was dependent on the rootstock used. Our results for $0^{\circ} \mathrm{C}$ storage and shelf life period were similar to those of Risse et al. (1990).

The effects of grafting on hallow heart were not significant during the storage and shelf life for both cultivars (Table 3 and 4 ). In similarly to our findings, it was reported that effect of rootstocks on hallow heart was not significant during shelf life by Özdemir et al. (2016). Cushman and Huan (2008) reported that a greater hollow heart ratio in non-grafted watermelon than in grafted watermelon. Moreover, it was reported the environmental and cultural conditions affect incidence of hollow heart beside to rootstocks by Özdemir et al. (2018).

The most abundant sugar was sucrose at the end of the storage time and shelf life in both cultivars (Table 5 and 6 ). Similar results were reported (Chisholm and Picha, 1986; Kyriacou and Soteriou, 2015; Özdemir et al., 2016, 2018). 
Table 3. The effects of rootstocks on citric acid (\%), malic acid (\%), external (rind) and internal (flesh) chilling injury (Cl, 15 ) and hallow heart (1-5) of Crisby (CR) watermelon fruits during storage at $0^{\circ} \mathrm{C}$ and following 7 days at $21^{\circ} \mathrm{C}$

\begin{tabular}{|c|c|c|c|c|c|c|c|c|c|c|c|}
\hline \multirow{2}{*}{ Parameters } & \multirow{2}{*}{ Scion/ rootstock } & \multicolumn{4}{|c|}{ Days in storage at $0^{\circ} \mathrm{C}$} & \multirow{2}{*}{ Mean } & \multicolumn{4}{|c|}{ Days in shelf life at $21^{\circ} \mathrm{C}$} & \multirow{2}{*}{ Mean } \\
\hline & & 0 & 7 & 14 & 21 & & $0+7$ & $7+7$ & $14+7$ & $21+7$ & \\
\hline \multirow{5}{*}{$\begin{array}{l}\text { Citric acid } \\
(\%)\end{array}$} & CR(Control) & $0.08 \mathrm{a}$ & $0.07 \mathrm{a}$ & $0.08 \mathrm{a}$ & $0.10 \mathrm{~b}$ & $0.08 \mathrm{~b}$ & $0.07 \mathrm{a}$ & $0.07 \mathrm{a}$ & $0.07 \mathrm{~b}$ & $0.08 \mathrm{~b}$ & $0.08 \mathrm{~b}$ \\
\hline & CR/Macis & $0.06 \mathrm{~b}$ & $0.06 \mathrm{a}$ & $0.08 \mathrm{a}$ & $0.07 \mathrm{c}$ & $0.07 \mathrm{c}$ & $0.07 \mathrm{a}$ & $0.06 \mathrm{a}$ & $0.07 \mathrm{~b}$ & $0.08 \mathrm{~b}$ & $.07 \mathrm{~b}$ \\
\hline & CR/Argentario & $.08 \mathrm{a}$ & $0.07 \mathrm{a}$ & $0.08 \mathrm{a}$ & $0.10 \mathrm{~b}$ & $0.08 \mathrm{~b}$ & $0.08 \mathrm{a}$ & $0.07 \mathrm{a}$ & $0.07 \mathrm{~b}$ & $0.07 \mathrm{~b}$ & \\
\hline & $\mathrm{CR}$ & $08 \mathrm{a}$ & $0.08 \mathrm{a}$ & $0.08 \mathrm{a}$ & $0.09 \mathrm{bc}$ & 0.08 & $0.08 \mathrm{a}$ & $0.07 \mathrm{a}$ & $0.10 \mathrm{a}$ & $0.11 \mathrm{a}$ & \\
\hline & & $09 \mathrm{a}$ & $0.08 \mathrm{a}$ & $0.09 \mathrm{a}$ & $0.13 \mathrm{a}$ & & $0.09 \mathrm{a}$ & $0.09 \mathrm{a}$ & $0.08 \mathrm{ab}$ & $0.11 \mathrm{a}$ & $9 \mathrm{a}$ \\
\hline \multirow{5}{*}{$\begin{array}{l}\text { Malic acid } \\
(\%)\end{array}$} & & $3 \mathrm{~b}$ & $22 \mathrm{a}$ & $0.25 \mathrm{~b}$ & $0.25 \mathrm{bc}$ & & $.25 \mathrm{a}$ & $0.20 \mathrm{~b}$ & $0.23 \mathrm{bc}$ & $0.25 \mathrm{bc}$ & $3 \mathrm{~b}$ \\
\hline & & $24 a b$ & $0.22 \mathrm{a}$ & $0.25 \mathrm{~b}$ & $0.24 \mathrm{c}$ & & $0.22 \mathrm{a}$ & $0.22 b$ & $0.23 \mathrm{bc}$ & $0.21 \mathrm{c}$ & \\
\hline & & $.19 \mathrm{c}$ & $0.19 a$ & $0.20 \mathrm{c}$ & $0.24 \mathrm{c}$ & 0.2 & $0.21 \mathrm{a}$ & $0.22 \mathrm{~b}$ & $0.21 \mathrm{c}$ & $0.23 \mathrm{~cd}$ & $.22 \mathrm{~b}$ \\
\hline & & $25 \mathrm{a}$ & $0.22 \mathrm{a}$ & $0.30 \mathrm{a}$ & $0.28 \mathrm{~b}$ & & $0.25 \mathrm{a}$ & $0.29 \mathrm{a}$ & $0.26 \mathrm{a}$ & $0.28 a b$ & $7 \mathrm{a}$ \\
\hline & & $24 a b$ & $0.22 \mathrm{a}$ & $0.25 \mathrm{~b}$ & $0.34 \mathrm{a}$ & 0.26 & $0.24 \mathrm{a}$ & $0.27 \mathrm{a}$ & $0.24 \mathrm{ab}$ & $0.29 \mathrm{a}$ & $0.26 \mathrm{a}$ \\
\hline \multirow{5}{*}{$\begin{array}{l}\text { Cl external } \\
(1-5)\end{array}$} & & $0 \mathrm{a}$ & $00 \mathrm{a}$ & $1.00 \mathrm{~b}$ & $1.46 \mathrm{a}$ & & $.00 \mathrm{a}$ & $1.00 \mathrm{a}$ & $1.29 \mathrm{a}$ & $1.23 \mathrm{a}$ & $3 a$ \\
\hline & & & $1.00 \mathrm{a}$ & $1.03 \mathrm{~b}$ & $1.61 \mathrm{a}$ & & $1.00 \mathrm{a}$ & $1.00 \mathrm{a}$ & $1.29 \mathrm{a}$ & $1.30 \mathrm{a}$ & $5 \mathrm{a}$ \\
\hline & & $1.00 \mathrm{a}$ & $1.00 \mathrm{a}$ & $1.00 \mathrm{~b}$ & $1.54 \mathrm{a}$ & $1.13 \mathrm{a}$ & $1.00 \mathrm{a}$ & $1.04 \mathrm{a}$ & $1.03 \mathrm{~b}$ & $1.29 \mathrm{a}$ & $1.09 \mathrm{a}$ \\
\hline & & $1.00 \mathrm{a}$ & $1.00 \mathrm{a}$ & $1.10 \mathrm{a}$ & $1.54 \mathrm{a}$ & & $1.00 \mathrm{a}$ & $1.04 \mathrm{a}$ & $1.20 a b$ & $1.66 \mathrm{a}$ & $1.23 \mathrm{a}$ \\
\hline & & $00 \mathrm{a}$ & $1.00 \mathrm{a}$ & $1.00 \mathrm{~b}$ & $1.57 \mathrm{a}$ & & $1.00 \mathrm{a}$ & $1.10 \mathrm{a}$ & $1.13 \mathrm{ab}$ & $1.21 \mathrm{a}$ & $1 \mathrm{a}$ \\
\hline \multirow{5}{*}{$\begin{array}{l}\mathrm{Cl} \text { internal } \\
(1-5)\end{array}$} & & $0 \mathrm{a}$ & $1.00 \mathrm{a}$ & $1.00 \mathrm{a}$ & $1.33 \mathrm{a}$ & & $1.00 \mathrm{a}$ & $1.00 \mathrm{a}$ & $1.07 \mathrm{a}$ & $1.00 \mathrm{a}$ & $2 a$ \\
\hline & & $0 \mathrm{a}$ & $1.00 \mathrm{a}$ & $1.00 \mathrm{a}$ & $1.00 \mathrm{a}$ & & $1.00 \mathrm{a}$ & $1.00 \mathrm{a}$ & $1.00 \mathrm{a}$ & $1.17 \mathrm{a}$ & $4 a$ \\
\hline & & $00 \mathrm{a}$ & $1.00 \mathrm{a}$ & $1.00 \mathrm{a}$ & $1.00 \mathrm{a}$ & $00 \mathrm{a}$ & $1.00 \mathrm{a}$ & $1.00 \mathrm{a}$ & $1.10 \mathrm{a}$ & $1.17 \mathrm{a}$ & $1.07 \mathrm{a}$ \\
\hline & & $00 \mathrm{a}$ & $1.00 \mathrm{a}$ & $1.00 \mathrm{a}$ & $1.00 \mathrm{a}$ & $1.00 \mathrm{a}$ & $1.00 \mathrm{a}$ & $1.00 \mathrm{a}$ & $1.00 \mathrm{a}$ & $1.33 \mathrm{a}$ & $1.08 \mathrm{a}$ \\
\hline & & $00 \mathrm{a}$ & $1.00 \mathrm{a}$ & $1.00 \mathrm{a}$ & $1.00 \mathrm{a}$ & & $1.00 \mathrm{a}$ & $1.00 \mathrm{a}$ & $1.00 \mathrm{a}$ & $1.00 \mathrm{a}$ & $1.00 \mathrm{a}$ \\
\hline \multirow{5}{*}{$\begin{array}{l}\text { Hallow } \\
\text { heart }(1-5)\end{array}$} & & $23 \mathrm{a}$ & $1.22 \mathrm{a}$ & $1.43 \mathrm{a}$ & $1.37 \mathrm{a}$ & & $1.23 \mathrm{a}$ & $1.53 \mathrm{a}$ & $1.50 \mathrm{a}$ & $1.43 \mathrm{a}$ & $1.43 \mathrm{a}$ \\
\hline & & $1.20 \mathrm{a}$ & $1.20 \mathrm{a}$ & $1.23 \mathrm{a}$ & $1.40 \mathrm{a}$ & $1.26 \mathrm{a}$ & $1.30 \mathrm{a}$ & $1.23 \mathrm{a}$ & $1.40 \mathrm{a}$ & $1.37 \mathrm{a}$ & $1.33 \mathrm{a}$ \\
\hline & $\mathrm{CR} /$ Argentario & $1.18 \mathrm{a}$ & $1.33 \mathrm{a}$ & $1.37 \mathrm{a}$ & $1.55 \mathrm{a}$ & $1.36 \mathrm{a}$ & $1.35 \mathrm{a}$ & $1.37 \mathrm{a}$ & $1.27 \mathrm{a}$ & $1.33 \mathrm{a}$ & $1.33 \mathrm{a}$ \\
\hline & CR/RS841 & $1.23 \mathrm{a}$ & $1.20 \mathrm{a}$ & $1.20 \mathrm{a}$ & $1.25 \mathrm{a}$ & $1.22 \mathrm{a}$ & $1.23 \mathrm{a}$ & $1.27 \mathrm{a}$ & $1.47 \mathrm{a}$ & $1.40 \mathrm{a}$ & $1.34 \mathrm{a}$ \\
\hline & CR/Ferro & $1.28 \mathrm{a}$ & $1.33 \mathrm{a}$ & $1.30 \mathrm{a}$ & $1.43 \mathrm{a}$ & $1.34 \mathrm{a}$ & $1.37 \mathrm{a}$ & $1.42 \mathrm{a}$ & $130 \mathrm{a}$ & $1.33 \mathrm{a}$ & $1.35 \mathrm{a}$ \\
\hline
\end{tabular}

${ }^{x}$ Mean separation was performed by Fisher's LSD test. Means $(n=3)$ followed by same letters within a column are not significantly different at $\mathrm{P}<0.05$.

Table 4. The effects of rootstocks on citric acid (\%), malic acid (\%), external (rind) and internal (flesh) chilling injury (Cl, 15) and hallow heart (1-5) of Crimson Tide (CT) watermelon fruits during storage at $0^{\circ} \mathrm{C}$ and following 7 days at $21^{\circ} \mathrm{C}$

\begin{tabular}{|c|c|c|c|c|c|c|c|c|c|c|c|}
\hline \multirow{2}{*}{ Parameters } & \multirow{2}{*}{ Scion/ rootstock } & \multicolumn{4}{|c|}{ Days in storage at $0^{\circ} \mathrm{C}$} & \multirow{2}{*}{ Mean } & \multicolumn{4}{|c|}{ Days in shelf life at $21^{\circ} \mathrm{C}$} & \multirow{2}{*}{ Mean } \\
\hline & & 0 & 7 & 14 & 21 & & $0+7$ & $7+7$ & $14+7$ & $21+7$ & \\
\hline \multirow{5}{*}{$\begin{array}{l}\text { Citric acid } \\
(\%)\end{array}$} & CT(Control) & $0.10 \mathrm{a}$ & $0.09 \mathrm{a}$ & $0.12 a b$ & $0.10 \mathrm{a}$ & $0.10 \mathrm{a}$ & $0.10 \mathrm{a}$ & $0.07 \mathrm{~b}$ & $0.08 \mathrm{a}$ & $0.07 \mathrm{a}$ & $0.08 \mathrm{~b}$ \\
\hline & CT/Macis & $0.06 \mathrm{~b}$ & $0.05 \mathrm{~b}$ & $0.09 \mathrm{~b}$ & $0.07 \mathrm{~b}$ & & $0.09 \mathrm{a}$ & $0.09 \mathrm{~b}$ & $0.05 \mathrm{a}$ & $0.08 \mathrm{a}$ & $0.08 \mathrm{~b}$ \\
\hline & CT/Argentario & $0.08 \mathrm{ab}$ & $0.08 \mathrm{ab}$ & $0.09 \mathrm{~b}$ & $0.07 \mathrm{~b}$ & $0.08 \mathrm{~b}$ & $0.09 \mathrm{a}$ & $0.08 \mathrm{~b}$ & $0.07 \mathrm{a}$ & $0.07 \mathrm{a}$ & \\
\hline & $\mathrm{CT} / \mathrm{P}$ & $0.10 \mathrm{a}$ & $0.09 \mathrm{a}$ & $0.14 \mathrm{a}$ & $0.09 a b$ & $0.10 \mathrm{a}$ & $0.10 \mathrm{a}$ & $0.14 \mathrm{a}$ & $0.07 \mathrm{a}$ & $0.09 \mathrm{a}$ & \\
\hline & & $10 \mathrm{a}$ & $0.09 \mathrm{a}$ & $0.13 a b$ & $0.09 \mathrm{a}$ & $0.10 \mathrm{a}$ & $0.09 \mathrm{a}$ & $0.12 \mathrm{a}$ & $0.09 \mathrm{a}$ & $0.10 \mathrm{a}$ & $0.10 \mathrm{~b}$ \\
\hline \multirow{5}{*}{$\begin{array}{l}\text { Malic acid } \\
(\%)\end{array}$} & & $3 \mathrm{c}$ & $0.23 \mathrm{~cd}$ & $0.25 \mathrm{~b}$ & $0.26 \mathrm{ab}$ & $4 c$ & $0.24 \mathrm{a}$ & $0.31 \mathrm{a}$ & $0.24 \mathrm{c}$ & $0.25 \mathrm{~b}$ & $6 \mathrm{bc}$ \\
\hline & & $21 \mathrm{c}$ & $0.21 d$ & $0.26 \mathrm{~b}$ & $0.20 \mathrm{~b}$ & $0.22 \mathrm{c}$ & $0.23 \mathrm{a}$ & $0.29 \mathrm{a}$ & $0.21 \mathrm{c}$ & $0.24 \mathrm{~b}$ & $0.24 c$ \\
\hline & $\mathrm{CT} / \mathrm{Ar}$ & $0.27 b$ & $0.26 \mathrm{bc}$ & $0.32 \mathrm{a}$ & $0.31 \mathrm{a}$ & $0.29 \mathrm{~b}$ & $0.25 \mathrm{a}$ & $0.29 \mathrm{a}$ & $0.26 \mathrm{bc}$ & $0.24 b$ & $0.26 \mathrm{bc}$ \\
\hline & & $30 \mathrm{a}$ & $0.29 a b$ & $0.36 \mathrm{a}$ & $0.32 \mathrm{a}$ & & $0.24 \mathrm{a}$ & $0.32 \mathrm{a}$ & $0.29 a b$ & $0.32 \mathrm{a}$ & \\
\hline & & $32 \mathrm{a}$ & $0.31 \mathrm{a}$ & $0.33 \mathrm{a}$ & $0.32 \mathrm{a}$ & & $0.25 a$ & $0.27 \mathrm{a}$ & $0.32 \mathrm{a}$ & $0.28 a b$ & $8 a b$ \\
\hline \multirow{5}{*}{$\begin{array}{l}\text { Cl external } \\
(1-5)\end{array}$} & & & $00 \mathrm{a}$ & & $1.20 \mathrm{a}$ & & $1.00 \mathrm{a}$ & $1.00 \mathrm{a}$ & $1.29 \mathrm{a}$ & $2.04 \mathrm{a}$ & $3 a$ \\
\hline & & $0 \mathrm{a}$ & $1.00 \mathrm{a}$ & $1.13 \mathrm{a}$ & $1.47 \mathrm{a}$ & & $1.00 \mathrm{a}$ & $1.13 \mathrm{a}$ & $1.29 \mathrm{a}$ & $2.13 \mathrm{a}$ & 9 a \\
\hline & $\mathrm{CT} / \mathrm{A}$ & $00 \mathrm{a}$ & $1.00 \mathrm{a}$ & $1.00 \mathrm{a}$ & $1.17 \mathrm{a}$ & $4 \mathrm{a}$ & $1.00 \mathrm{a}$ & $1.00 \mathrm{a}$ & $1.17 \mathrm{a}$ & $2.23 \mathrm{a}$ & $5 a$ \\
\hline & & $00 \mathrm{a}$ & $1.00 \mathrm{a}$ & $1.11 \mathrm{a}$ & $1.25 \mathrm{a}$ & & $1.00 \mathrm{a}$ & $1.13 \mathrm{a}$ & $1.13 \mathrm{a}$ & $2.61 \mathrm{a}$ & $7 \mathrm{a}$ \\
\hline & & $0 \mathrm{a}$ & $1.00 \mathrm{a}$ & $4 a$ & $1.29 \mathrm{a}$ & & $1.00 \mathrm{a}$ & $1.13 \mathrm{a}$ & $1.13 \mathrm{a}$ & $2.33 \mathrm{a}$ & $1.40 \mathrm{a}$ \\
\hline \multirow{5}{*}{$\begin{array}{l}\mathrm{Cl} \text { internal } \\
(1-5)\end{array}$} & & $0 \mathrm{a}$ & $1.00 \mathrm{a}$ & $0 \mathrm{a}$ & $1.00 \mathrm{a}$ & & $1.00 \mathrm{a}$ & $1.00 \mathrm{a}$ & $1.00 \mathrm{a}$ & $2.09 \mathrm{a}$ & $1.27 \mathrm{a}$ \\
\hline & $\mathrm{CT} / \mathrm{I}$ & $0 \mathrm{a}$ & $1.00 \mathrm{a}$ & $1.00 \mathrm{a}$ & $1.00 \mathrm{a}$ & $0 \mathrm{a}$ & $1.00 \mathrm{a}$ & $1.00 \mathrm{a}$ & $1.00 \mathrm{a}$ & $1.00 \mathrm{a}$ & $1.00 \mathrm{a}$ \\
\hline & $\mathrm{CT} / \mathrm{l}$ & $00 \mathrm{a}$ & $1.00 \mathrm{a}$ & $1.00 \mathrm{a}$ & $1.00 \mathrm{a}$ & $1.00 \mathrm{a}$ & $1.00 \mathrm{a}$ & $1.00 \mathrm{a}$ & $1.00 \mathrm{a}$ & $1.13 \mathrm{a}$ & $1.03 \mathrm{a}$ \\
\hline & CT/ & $1.00 \mathrm{a}$ & $1.00 \mathrm{a}$ & $1.00 \mathrm{a}$ & $1.00 \mathrm{a}$ & $1.00 \mathrm{a}$ & $1.00 \mathrm{a}$ & $1.00 \mathrm{a}$ & $1.00 \mathrm{a}$ & $1.00 \mathrm{a}$ & $1.00 \mathrm{a}$ \\
\hline & & $00 \mathrm{a}$ & $1.00 \mathrm{a}$ & & $1.00 \mathrm{a}$ & & $1.00 \mathrm{a}$ & $1.00 \mathrm{a}$ & $1.00 \mathrm{a}$ & $1.00 \mathrm{a}$ & $1.00 \mathrm{a}$ \\
\hline \multirow{5}{*}{$\begin{array}{l}\text { Hallow } \\
\text { heart (1- } \\
5)\end{array}$} & & $13 a$ & $1.42 \mathrm{a}$ & & $1.27 \mathrm{a}$ & & $1.63 \mathrm{a}$ & $1.21 \mathrm{a}$ & $1.38 \mathrm{a}$ & $1.31 \mathrm{a}$ & $1.38 \mathrm{a}$ \\
\hline & CT/Macis & $03 a$ & $1.50 \mathrm{a}$ & $1.50 \mathrm{a}$ & $1.25 \mathrm{a}$ & $1.32 \mathrm{a}$ & $1.21 \mathrm{a}$ & $1.21 \mathrm{a}$ & $1.13 \mathrm{a}$ & $1.42 \mathrm{a}$ & $1.24 \mathrm{a}$ \\
\hline & CT/Argentario & $1.10 \mathrm{a}$ & $1.21 \mathrm{a}$ & $1.21 \mathrm{a}$ & $1.08 \mathrm{a}$ & $1.15 \mathrm{a}$ & $1.09 \mathrm{a}$ & $1.04 \mathrm{a}$ & $1.29 \mathrm{a}$ & $1.49 \mathrm{a}$ & $1.23 \mathrm{a}$ \\
\hline & CT/RS841 & $1.37 \mathrm{a}$ & $1.17 \mathrm{a}$ & $1.23 \mathrm{a}$ & $1.17 \mathrm{a}$ & $1.24 \mathrm{a}$ & $1.14 \mathrm{a}$ & $1.29 \mathrm{a}$ & $1.17 \mathrm{a}$ & $1.38 \mathrm{a}$ & $1.25 \mathrm{a}$ \\
\hline & CT/Ferro & $1.30 \mathrm{a}$ & $1.71 \mathrm{a}$ & $1.08 \mathrm{a}$ & $1.34 \mathrm{a}$ & $1.36 \mathrm{a}$ & $1.09 \mathrm{a}$ & $1.21 \mathrm{a}$ & $1.13 \mathrm{a}$ & $1.07 \mathrm{a}$ & $1.12 \mathrm{a}$ \\
\hline
\end{tabular}

${ }^{x}$ Mean separation was performed by Fisher's LSD test. Means $(n=3)$ followed by same letters within a column are not significantly different at $\mathrm{P}<0.05$. 


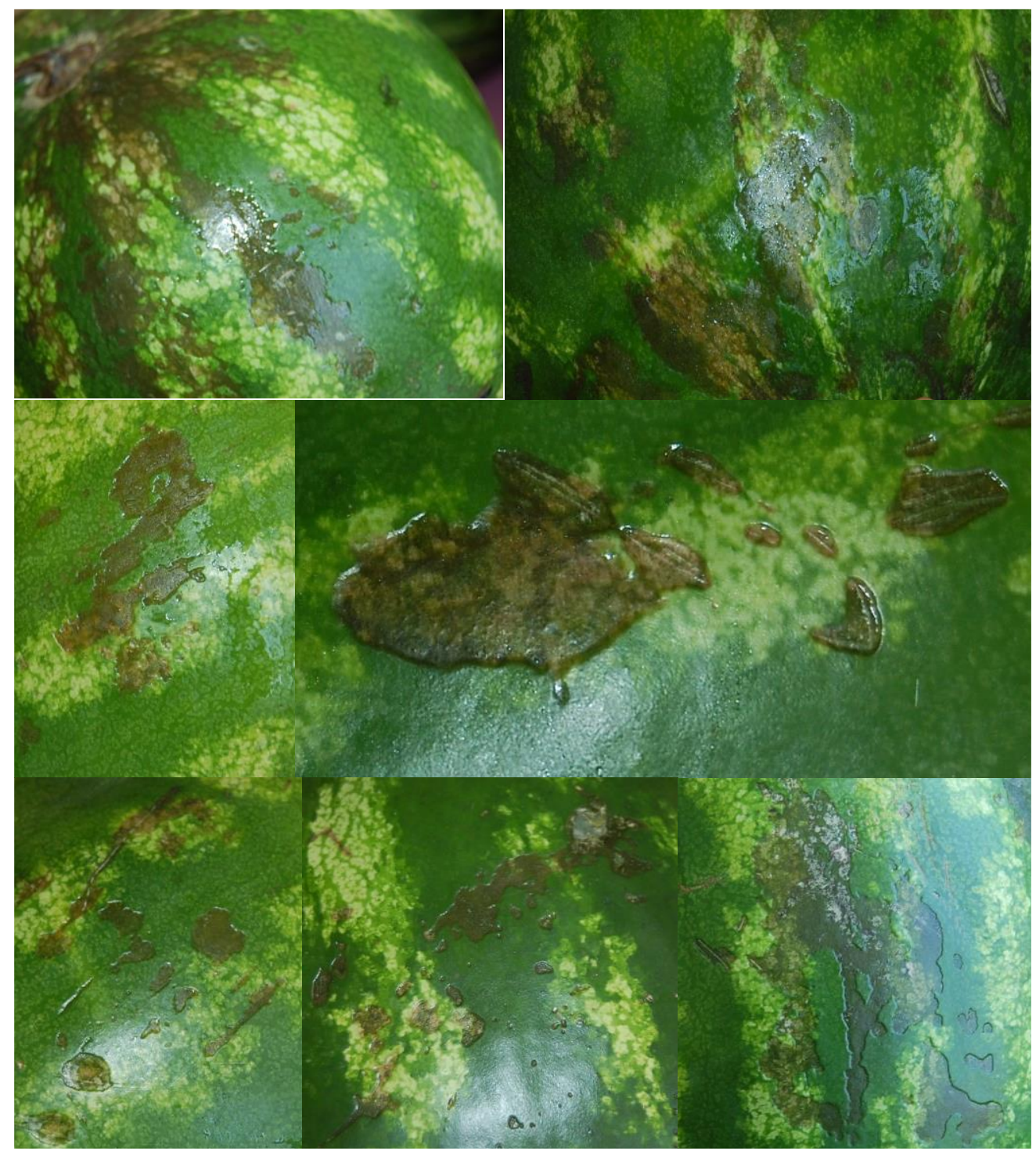

Figure 7. Rind $\mathrm{Cl}$ on fruit grafted on RS841 (above), Ferro (in the middle) and Argentario (below) rootstocks after 21+7 days of shelf life in CT cultivar

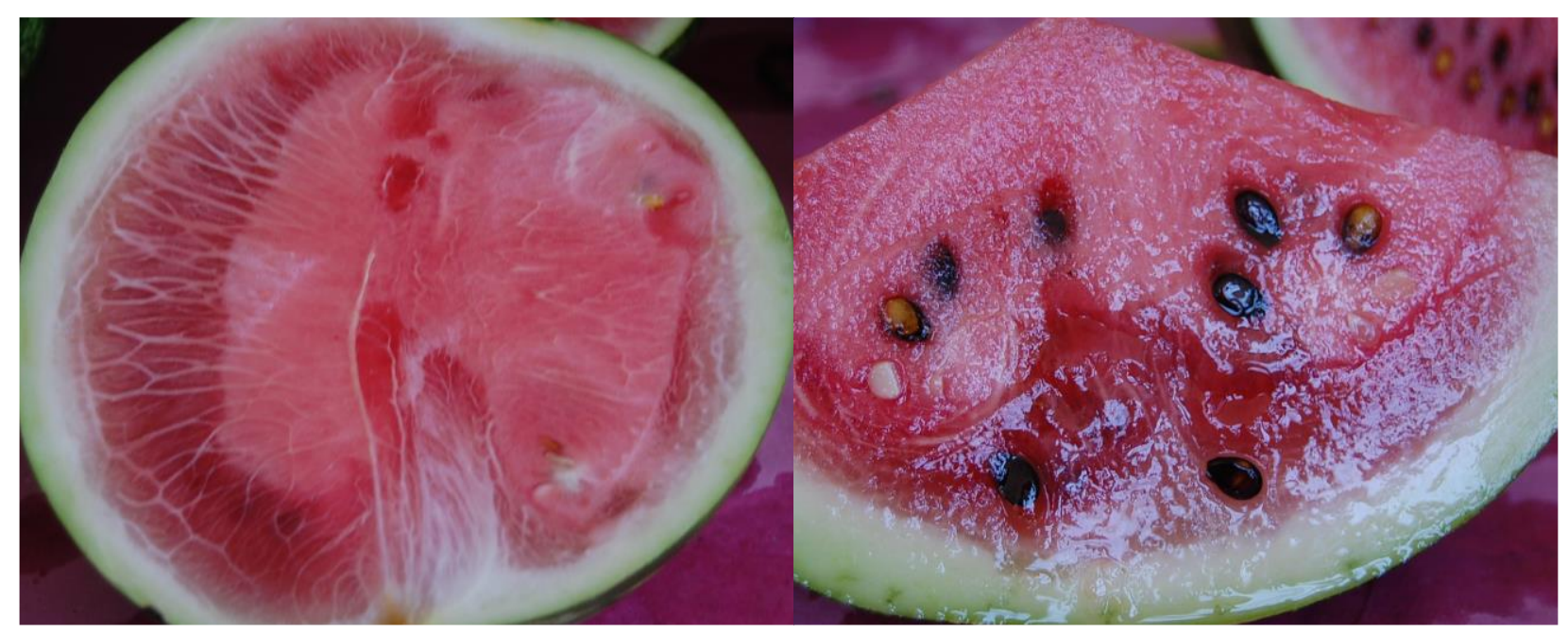

Figure 8. Flesh $\mathrm{Cl}$ on fruit grafted on Macis (left) rootstock and control fruits (right) after 21+7 days of shelf life in CR cultivar 
Table 5. The effects of rootstocks on fructose (\%), glucose (\%), sucrose (\%) total sugar (\%) and sensory quality (1-9) of Crisby $(\mathrm{CR})$ watermelon fruits during storage at $0^{\circ} \mathrm{C}$ and following 7 days at $21^{\circ} \mathrm{C}$

\begin{tabular}{|c|c|c|c|c|c|c|c|c|c|c|c|}
\hline \multirow{2}{*}{ Parameters } & \multirow{2}{*}{ Scion/ rootstock } & \multicolumn{4}{|c|}{ Days in storage at $0^{\circ} \mathrm{C}$} & \multirow{2}{*}{ Mean } & \multicolumn{4}{|c|}{ Days in shelf life at $21^{\circ} \mathrm{C}$} & \multirow{2}{*}{ Mean } \\
\hline & & 0 & 7 & 14 & 21 & & $0+7$ & $7+7$ & $14+7$ & $21+7$ & \\
\hline \multirow{5}{*}{$\begin{array}{l}\text { Fructose } \\
(\%)\end{array}$} & CR(Control) & $4.21 \mathrm{a}$ & $3.94 \mathrm{a}$ & $3.15 \mathrm{a}$ & $3.32 \mathrm{bc}$ & $3.65 \mathrm{a}$ & $3.21 \mathrm{a}$ & $3.07 \mathrm{a}$ & $3.09 \mathrm{a}$ & $3.42 \mathrm{a}$ & $3.20 \mathrm{a}$ \\
\hline & R/Macis & $4.27 \mathrm{a}$ & $4.03 \mathrm{a}$ & $3.36 \mathrm{a}$ & $3.16 \mathrm{c}$ & $3.70 \mathrm{a}$ & $3.22 \mathrm{a}$ & $3.50 \mathrm{a}$ & $3.12 \mathrm{a}$ & $2.84 \mathrm{a}$ & \\
\hline & $\mathrm{CR} / \mathrm{Arg}$ & $4.11 \mathrm{a}$ & $3.75 \mathrm{a}$ & $3.27 \mathrm{a}$ & $3.25 \mathrm{bc}$ & $3.60 \mathrm{a}$ & $2.94 \mathrm{ab}$ & $3.28 \mathrm{a}$ & $3.26 \mathrm{a}$ & $3.32 \mathrm{a}$ & \\
\hline & & $67 \mathrm{a}$ & $3.61 \mathrm{a}$ & $3.56 \mathrm{a}$ & $3.68 a b$ & $3.63 \mathrm{a}$ & $3.15 \mathrm{a}$ & $3.37 \mathrm{a}$ & $3.43 \mathrm{a}$ & $3.45 \mathrm{a}$ & \\
\hline & C & $05 \mathrm{a}$ & $3.88 a$ & 3.58 & $3.96 \mathrm{a}$ & 3.87 & $2.73 \mathrm{~b}$ & $3.60 \mathrm{a}$ & $3.31 \mathrm{a}$ & $3.66 \mathrm{a}$ & \\
\hline \multirow{5}{*}{$\begin{array}{l}\text { Glucose } \\
(\%)\end{array}$} & & $59 \mathrm{a}$ & $2.32 \mathrm{a}$ & $1.90 \mathrm{a}$ & $2.13 \mathrm{a}$ & & $1.66 \mathrm{a}$ & $1.60 \mathrm{a}$ & $1.72 \mathrm{a}$ & $1.87 \mathrm{ab}$ & \\
\hline & & $74 \mathrm{a}$ & $2.52 \mathrm{a}$ & $1.94 \mathrm{a}$ & $1.94 \mathrm{a}$ & & $1.66 \mathrm{a}$ & $1.54 \mathrm{a}$ & $1.78 \mathrm{a}$ & $1.61 \mathrm{~b}$ & \\
\hline & & $2.62 \mathrm{a}$ & $2.30 \mathrm{a}$ & $2.05 \mathrm{a}$ & $2.13 \mathrm{a}$ & $2.28 \mathrm{a}$ & $1.55 \mathrm{a}$ & $1.86 \mathrm{a}$ & $1.80 \mathrm{a}$ & $1.90 \mathrm{ab}$ & 1.78 \\
\hline & & $27 \mathrm{a}$ & $2.23 \mathrm{a}$ & $2.21 \mathrm{a}$ & $2.33 \mathrm{a}$ & 2.2 & $1.54 \mathrm{a}$ & $1.61 \mathrm{a}$ & $1.96 \mathrm{a}$ & $1.92 \mathrm{a}$ & \\
\hline & & $43 \mathrm{a}$ & $2.28 a$ & $2.31 \mathrm{a}$ & $2.48 \mathrm{a}$ & 2.3 & $1.32 \mathrm{a}$ & $1.90 \mathrm{a}$ & $1.85 \mathrm{a}$ & $2.12 \mathrm{a}$ & \\
\hline \multirow{5}{*}{$\begin{array}{l}\text { Sucrose } \\
(\%)\end{array}$} & & $7 \mathrm{a}$ & 3.58 & $.84 \mathrm{a}$ & $3.49 \mathrm{a}$ & $3.72 \mathrm{a}$ & $4.47 \mathrm{a}$ & $4.07 \mathrm{a}$ & $4.34 \mathrm{a}$ & $4.60 \mathrm{a}$ & \\
\hline & & $76 a$ & 3.40 & $4.00 \mathrm{a}$ & $3.54 \mathrm{a}$ & $3.67 \mathrm{a}$ & $4.85 \mathrm{a}$ & $3.95 \mathrm{a}$ & $4.18 \mathrm{a}$ & $4.14 \mathrm{a}$ & $B a$ \\
\hline & & $84 \mathrm{a}$ & $3.63 \mathrm{a}$ & $3.80 \mathrm{a}$ & $3.85 \mathrm{a}$ & $3.78 \mathrm{a}$ & $5.27 \mathrm{a}$ & $4.26 \mathrm{a}$ & $4.01 \mathrm{a}$ & $4.56 \mathrm{a}$ & $4.53 \mathrm{a}$ \\
\hline & & $17 \mathrm{a}$ & $3.98 \mathrm{a}$ & $3.29 \mathrm{a}$ & $3.16 \mathrm{a}$ & $3.65 \mathrm{a}$ & $4.72 \mathrm{a}$ & $4.01 \mathrm{a}$ & $3.81 \mathrm{a}$ & $4.23 \mathrm{a}$ & 9 a \\
\hline & & $77 \mathrm{a}$ & 3.63 & $3.50 \mathrm{a}$ & $4.44 \mathrm{a}$ & & $4.94 \mathrm{a}$ & $3.98 \mathrm{a}$ & $4.25 \mathrm{a}$ & $4.46 \mathrm{a}$ & \\
\hline \multirow{5}{*}{$\begin{array}{l}\text { Total sugar } \\
\text { (\%) }\end{array}$} & & $76 \mathrm{a}$ & 9.83 & $8.88 \mathrm{a}$ & $8.94 \mathrm{~b}$ & & $9.34 \mathrm{a}$ & $8.73 \mathrm{a}$ & $9.14 \mathrm{a}$ & $9.88 \mathrm{a}$ & \\
\hline & & $76 \mathrm{a}$ & 9.94 & 9.2 & $8.63 \mathrm{~b}$ & $9.66 \mathrm{a}$ & $9.73 \mathrm{a}$ & $8.99 \mathrm{a}$ & $9.07 \mathrm{a}$ & $8.59 \mathrm{a}$ & $9 a$ \\
\hline & & $.56 \mathrm{a}$ & $9.68 \mathrm{a}$ & $9.12 \mathrm{a}$ & $9.23 \mathrm{~b}$ & $9.65 \mathrm{a}$ & $9.76 \mathrm{a}$ & $9.40 \mathrm{a}$ & $9.07 \mathrm{a}$ & $9.77 \mathrm{a}$ & $9.50 \mathrm{a}$ \\
\hline & & $0.10 \mathrm{a}$ & 9.82 a & $9.05 \mathrm{a}$ & 9.17 b & $9.53 \mathrm{a}$ & $9.40 \mathrm{a}$ & $8.99 \mathrm{a}$ & $9.20 \mathrm{a}$ & $9.59 \mathrm{a}$ & $9.29 \mathrm{a}$ \\
\hline & & $0.24 \mathrm{a}$ & $9.78 \mathrm{a}$ & $9.39 \mathrm{a}$ & $10.88 \mathrm{a}$ & $10.07 \mathrm{a}$ & $8.97 \mathrm{a}$ & $9.48 \mathrm{a}$ & $9.41 \mathrm{a}$ & $10.23 \mathrm{a}$ & $9.52 \mathrm{a}$ \\
\hline \multirow{5}{*}{$\begin{array}{l}\text { Sensory } \\
\text { quality } \\
(1-9)\end{array}$} & & $30 \mathrm{a}$ & $8.30 a$ & $7.00 \mathrm{~b}$ & $5.40 \mathrm{~b}$ & 7.3 & $8.10 \mathrm{a}$ & $7.10 \mathrm{c}$ & $6.50 \mathrm{c}$ & $5.50 \mathrm{c}$ & $6.80 \mathrm{c}$ \\
\hline & & $40 \mathrm{a}$ & $8.40 \mathrm{a}$ & $7.80 \mathrm{a}$ & $6.60 \mathrm{~b}$ & $7.80 \mathrm{a}$ & $8.00 \mathrm{a}$ & $7.60 \mathrm{ab}$ & $6.80 \mathrm{bc}$ & $5.80 \mathrm{~b}$ & $7.10 \mathrm{~b}$ \\
\hline & CR/Argentario & $8.00 \mathrm{a}$ & $8.30 \mathrm{a}$ & $7.20 \mathrm{~b}$ & $6.90 \mathrm{a}$ & $7.60 \mathrm{ab}$ & $8.10 \mathrm{a}$ & $8.00 \mathrm{ab}$ & $6.90 \mathrm{bc}$ & $6.10 \mathrm{~b}$ & $7.30 \mathrm{ab}$ \\
\hline & CR/RS841 & $8.20 \mathrm{a}$ & $8.60 \mathrm{a}$ & $8.00 \mathrm{a}$ & $7.00 \mathrm{a}$ & $7.90 \mathrm{a}$ & $8.20 \mathrm{a}$ & $7.50 \mathrm{bc}$ & $7.00 a b$ & $6.80 \mathrm{a}$ & $7.40 \mathrm{a}$ \\
\hline & CR/Ferro & $8.20 \mathrm{a}$ & $8.70 \mathrm{a}$ & $7.80 \mathrm{a}$ & $7.20 \mathrm{a}$ & $8.00 \mathrm{a}$ & $8.40 \mathrm{a}$ & $8.10 \mathrm{a}$ & $7.30 \mathrm{a}$ & $6.30 \mathrm{a} \mathrm{b}$ & $7.50 \mathrm{a}$ \\
\hline
\end{tabular}

${ }^{x}$ Mean separation was performed by Fisher's LSD test. Means $(n=3)$ followed by same letters within a column are not significantly different at $\mathrm{P}<0.05$.

Table 6. The effects of rootstocks on fructose (\%), glucose (\%), sucrose (\%) total sugar (\%) and sensory quality (1-9) of Crimson Tide (CT) watermelon fruits during storage at $0^{\circ} \mathrm{C}$ and following 7 days at $21^{\circ} \mathrm{C}$

\begin{tabular}{|c|c|c|c|c|c|c|c|c|c|c|c|}
\hline \multirow{2}{*}{ Parameters } & \multirow{2}{*}{ Scion/ rootstock } & \multicolumn{4}{|c|}{ Days in storage at $0^{\circ} \mathrm{C}$} & \multirow{2}{*}{ Mean } & \multicolumn{4}{|c|}{ Days in shelf life at $21^{\circ} \mathrm{C}$} & \multirow{2}{*}{ Mean } \\
\hline & & 0 & 7 & 14 & 21 & & $0+7$ & $7+7$ & $14+7$ & $21+7$ & \\
\hline \multirow{5}{*}{$\begin{array}{l}\text { Fructose } \\
(\%)\end{array}$} & $\mathrm{CT}(\mathrm{C}$ & $2.89 \mathrm{c}$ & $2.82 \mathrm{~b}$ & $2.54 \mathrm{c}$ & $3.33 \mathrm{ab}$ & $2.90 \mathrm{c}$ & $2.81 \mathrm{bc}$ & $3.07 \mathrm{a}$ & $3.10 \mathrm{ab}$ & $3.59 \mathrm{a}$ & \\
\hline & & $.92 \mathrm{bc}$ & $2.91 \mathrm{~b}$ & $3.09 \mathrm{~b}$ & $2.51 \mathrm{~b}$ & & $2.63 c$ & $3.14 \mathrm{a}$ & $2.73 b$ & $3.12 \mathrm{a}$ & \\
\hline & & & $3.13 a b$ & $3.48 a b$ & $3.26 a b$ & & $3.14 a b$ & $3.42 \mathrm{a}$ & $2.83 \mathrm{~b}$ & $2.96 \mathrm{a}$ & \\
\hline & & & & $9 \mathrm{a}$ & $3.58 \mathrm{a}$ & & $3.01 \mathrm{ab}$ & $3.54 \mathrm{a}$ & $2.86 \mathrm{~b}$ & $3.44 \mathrm{a}$ & \\
\hline & & $58 \mathrm{a}$ & $3.46 \mathrm{a}$ & $3.35 a b$ & $3.71 \mathrm{a}$ & & $3.16 \mathrm{a}$ & $3.12 \mathrm{a}$ & $3.45 \mathrm{a}$ & $3.16 \mathrm{a}$ & \\
\hline \multirow{5}{*}{$\begin{array}{l}\text { Glucose } \\
(\%)\end{array}$} & & $38 \mathrm{c}$ & $1.80 \mathrm{~b}$ & $1.71 \mathrm{c}$ & $2.15 \mathrm{ab}$ & & $1.79 \mathrm{a}$ & $1.81 \mathrm{a}$ & $1.70 \mathrm{a}$ & $2.13 \mathrm{a}$ & \\
\hline & & $b$ & & $1.99 \mathrm{bc}$ & $1.57 \mathrm{c}$ & & $1.75 \mathrm{a}$ & $1.88 \mathrm{a}$ & $1.55 \mathrm{a}$ & $1.83 \mathrm{a}$ & \\
\hline & & & $1.97 \mathrm{~b}$ & $2.06 a b$ & $1.87 \mathrm{bc}$ & & $1.73 \mathrm{a}$ & $1.89 \mathrm{a}$ & $1.49 \mathrm{a}$ & $1.62 \mathrm{a}$ & \\
\hline & & & $2.41 \mathrm{a}$ & $2.36 \mathrm{a}$ & $2.22 a b$ & & $1.86 \mathrm{a}$ & $2.20 \mathrm{a}$ & $1.57 \mathrm{a}$ & $2.01 \mathrm{a}$ & \\
\hline & & $42 \mathrm{a}$ & $2.34 \mathrm{a}$ & $2.19 \mathrm{ab}$ & $2.38 \mathrm{a}$ & & $1.88 \mathrm{a}$ & $1.80 \mathrm{a}$ & $1.75 \mathrm{a}$ & $1.80 \mathrm{a}$ & \\
\hline \multirow{5}{*}{$\begin{array}{l}\text { Sucrose } \\
(\%)\end{array}$} & & $24 a$ & $4.72 \mathrm{a}$ & $5.15 \mathrm{a}$ & $4.32 \mathrm{a}$ & & $4.90 \mathrm{a}$ & $5.60 \mathrm{ab}$ & $5.31 \mathrm{a}$ & $4.41 \mathrm{a}$ & \\
\hline & & $70 \mathrm{~b}$ & $4.35 \mathrm{a}$ & $4.36 \mathrm{a}$ & $4.85 \mathrm{a}$ & & $4.47 \mathrm{a}$ & $6.39 \mathrm{a}$ & $4.61 \mathrm{a}$ & $5.16 \mathrm{a}$ & \\
\hline & & & $4.66 \mathrm{a}$ & $4.22 \mathrm{a}$ & $4.54 \mathrm{a}$ & & $4.35 \mathrm{a}$ & $4.68 \mathrm{bc}$ & $5.27 \mathrm{a}$ & $4.94 \mathrm{a}$ & \\
\hline & & & $a$ & $4.31 \mathrm{a}$ & $3.88 \mathrm{a}$ & & $4.86 \mathrm{a}$ & bc & $4.59 \mathrm{a}$ & $5.07 \mathrm{a}$ & \\
\hline & & $34 \mathrm{bc}$ & $0 \mathrm{a}$ & $4.82 \mathrm{a}$ & $4.35 \mathrm{a}$ & & $4.70 \mathrm{a}$ & $3.98 c$ & $5.55 \mathrm{a}$ & $4.31 \mathrm{a}$ & $\mathrm{ta}$ \\
\hline \multirow{5}{*}{$\begin{array}{l}\text { Total } \\
\text { sugar (\%) }\end{array}$} & & $81 \mathrm{~cd}$ & $9.34 \mathrm{a}$ & $9.40 \mathrm{a}$ & $9.79 \mathrm{a}$ & $8 \mathrm{~cd}$ & $9.50 \mathrm{a}$ & $10.48 \mathrm{a}$ & $10.10 \mathrm{a}$ & $10.12 \mathrm{a}$ & $5 a$ \\
\hline & & $70 \mathrm{~d}$ & $9.25 \mathrm{a}$ & $9.44 \mathrm{a}$ & $8.92 \mathrm{a}$ & & $8.84 \mathrm{a}$ & $11.40 \mathrm{a}$ & $8.89 a$ & $10.10 \mathrm{a}$ & \\
\hline & & $.16 \mathrm{c}$ & $9.75 \mathrm{a}$ & $9.76 \mathrm{a}$ & $9.67 \mathrm{a}$ & & $9.22 \mathrm{a}$ & $9.98 \mathrm{a}$ & $9.60 \mathrm{a}$ & $9.52 \mathrm{a}$ & \\
\hline & & $55 \mathrm{a}$ & $9.77 \mathrm{a}$ & $10.25 \mathrm{a}$ & $9.68 \mathrm{a}$ & $6 a b$ & $9.73 \mathrm{a}$ & $10.74 \mathrm{a}$ & $9.02 \mathrm{a}$ & $10.52 \mathrm{a}$ & $0 \mathrm{a}$ \\
\hline & & $.48 a b$ & $9.70 \mathrm{a}$ & $10.35 \mathrm{a}$ & $10.44 \mathrm{a}$ & & $9.73 \mathrm{a}$ & & $10.73 \mathrm{a}$ & $9.27 \mathrm{a}$ & $9.66 \mathrm{a}$ \\
\hline \multirow{5}{*}{$\begin{array}{l}\text { Sensory } \\
\text { quality } \\
(1-9)\end{array}$} & & $20 a b$ & $8.00 \mathrm{a}$ & $7.60 \mathrm{a}$ & $7.10 \mathrm{~b}$ & $7.70 \mathrm{bc}$ & $7.60 \mathrm{~b}$ & $6.60 \mathrm{~b}$ & $6.40 \mathrm{c}$ & $3.90 \mathrm{c}$ & $6.10 \mathrm{c}$ \\
\hline & & 7.90 b & $8.00 \mathrm{a}$ & $7.30 \mathrm{~b}$ & $7.20 \mathrm{~b}$ & $7.60 \mathrm{c}$ & $7.90 \mathrm{~b}$ & $7.20 \mathrm{ab}$ & $6.90 \mathrm{bc}$ & $5.00 \mathrm{~b}$ & $0 \mathrm{~b}$ \\
\hline & & & & & $7.00 \mathrm{~b}$ & & & & & $5.90 \mathrm{a}$ & \\
\hline & & & $8.30 \mathrm{a}$ & $7.90 \mathrm{a}$ & $8.10 \mathrm{a}$ & $8.20 \mathrm{a}$ & $8.20 \mathrm{a}$ & $7.30 a b$ & $7.00 \mathrm{ab}$ & $6.60 \mathrm{a}$ & $7.30 \mathrm{a}$ \\
\hline & CT/Ferro & $8.40 \mathrm{a}$ & $8.30 \mathrm{a}$ & $7.90 \mathrm{a}$ & $8.20 \mathrm{a}$ & $8.20 \mathrm{a}$ & $8.30 \mathrm{a}$ & $7.60 \mathrm{a}$ & $7.50 \mathrm{a}$ & $6.20 \mathrm{a}$ & $7.40 \mathrm{a}$ \\
\hline
\end{tabular}

${ }^{x}$ Mean separation was performed by Fisher's LSD test. Means $(n=3)$ followed by same letters within a column are not significantly different at $\mathrm{P}<0.05$. 
Changes in on fructose, glucose, sucrose, and total sugar contents were not significant during storage and shelf life in CR cultivar (Table 5).

In CT cultivar, fructose, glucose and total sugar contents were higher in fruit grafted on RS841 and Ferro rootstocks than those on other grafted and control fruit after 21 days of storage at $0^{\circ} \mathrm{C}$ and sucrose content was lower in fruit grafted on RS841 and Ferro rootstocks than other grafted and control fruit after 21 days of storage at $0^{\circ} \mathrm{C}$ (Table 6). Changes in on fructose, glucose, sucrose, and total sugar contents were not significant during shelf life in CT cultivar (Table 6). In previous studies, it was reported an accumulation of sucrose accompanied the decline in total soluble carbohydrates and soluble solids content in grafted and non-grafted watermelons during storage for 14 days at $25^{\circ} \mathrm{C}$ (Kyriacou and Soteriou, 2015) and Radulovic et al. (2007) reported that a significant decrease total sugar contents of watermelons during storage for 14 days at $20^{\circ} \mathrm{C}$. In contrast to our findings, Chisholm and Picha (1986) reported that sucrose, glucose, and fructose concentrations of watermelons mostly did not change during storage for 14 days at $0^{\circ} \mathrm{C}$ plus 5 days at $23^{\circ} \mathrm{C}$, but all generally were reduced at higher storage temperatures. Preservation of sugars at lower storage temperature may be attributed to a presumably lower rate of respiration (Özdemir et al., 2016). In similarly to our findings, in previous studies, between the hybrid rootstocks, mean sucrose concentration was undifferentiated (Kyriacou and Soteriou, 2015). Changes in on total and individual sugar contents were not significant during storage and shelf life (Özdemir et al., 2016). In CR cultivar, effect of grafting on total and individual sugar contents was not significant during storage (Özdemir et al., 2018). In one study, all of the sugars amounts in Crimson Tide watermelon fruit of grafting on the bottle gourd rootstocks enhancement compared to the control fruits and other rootstocks (Çandir et al., 2013). Lower sugar content was reported in grafted watermelon fruit than nongrafted fruit in some studies (Yetişir et al., 2003; Davis and Perkins-Veazie, 2005).

Taste scores (1-9) declined to the lowest level for 21 days of storage at $0^{\circ} \mathrm{C}$ in CR cultivar (Table 5 ) and the lowest level during shelf life in CT cultivar (Table 6). Lower taste score may be related to becoming of overripe of control fruit and grafted fruit on Macis and Argentario rootstocks and control fruit. Furthermore, panellists did not detect off-flavors in fruit from grafted plants. As the storage time extended, taste tented to decrease, taste scores of 7.9-8.5 were given to the fruit, which was initially tested by the tasting panellists and decreased to mean scores of $>6.1-7.5$ during storage at $0^{\circ} \mathrm{C}$ for 21 days and additional 7 days shelf life at $21^{\circ} \mathrm{C}$. But, In CT cultivar, taste scores of control fruit with 3.90 scores decreased to the lowest level at the third week of shelf life. This taste scores in control fruit were found below the acceptability $(>5.00)$ limit (Table 6). In CR cultivar, all grafted fruit higher taste scores than control fruit after 21 days of storage (Table 5). In CT cultivar, fruit grafted on Ferro, RS841 and Argentario rootstocks received higher taste scores than those on Macis rootstock and control fruit after 21 days of storage (Table 6). Bruton et al. (2009) and Özdemir et al. (2016, 2018) reported similar findings with the fruit from grafted watermelons.

Effects of grafting on flesh color lightness $\left(L^{*}\right.$ value) was not significant at the end of the storage time and additional 7 days shelf life at $21^{\circ} \mathrm{C}$ for both cultivars (Table 7 and 8). In contrast to our findings, in previous studies, flesh color lightness decreased during storage and/or shelf life in CR and CT cultivar Özdemir et al. (2016, 2018). Perkins-Veazie and Collins (2006) determined lower flesh color $L^{*}$ values in the fruit after 14 days of storage at $21^{\circ} \mathrm{C}$, compared freshly harvested watermelons. Kyriacou and Soteriou (2015) reported that flesh color lightness of watermelon fruit was affected by rootstock and storage and all hybrid rootstocks invariably maintained darker flesh color during storage.

In CR cultivar, flesh color $\mathrm{C}^{*}$ value peaked after 7 days and then decreased during storage (Table 7). In CT cultivar, flesh color $C^{*}$ value showed gradual decrease toward the end of storage (Table 8). In CR fruit, fruit grafted on RS841 and Ferro rootstocks had more compact (higher $\mathrm{C}^{*}$ ) color than those on other rootstocks and control fruit during storage and shelf life (Table 7). In CT fruit, during the storage, fruit grafted on Ferro, RS841 and Argentario rootstocks had higher flesh color $\mathrm{C}^{*}$ value than control fruit and grafted on Macis rootstock.

The effect of rootstocks on flesh color $C^{*}$ value was not significant during shelf life fruit grafted on Ferro, RS841 and Argentario rootstocks had higher flesh color $C^{*}$ value than those on Macis and control fruit during storage and effect of rootstocks on flesh color $\mathrm{C}^{*}$ value was not significant during shelf life (Table 8). In similarly to our findings in CR cultivar, Özdemir et al. (2016) reported that flesh color C* value continuously decreased during shelf life period at $21^{\circ} \mathrm{C}$ following storage at $7^{\circ} \mathrm{C}$ in $\mathrm{CR}$ and CT (except our findings in CT cultivar). Özdemir et al. (2018) reported similar findings with the fruit from grafted watermelons.

The flesh color $h^{\circ}$ values showed a progressive increase in non-grafted fruit with a lesser extent in grafted fruit during storage in both cultivars (Table 7 and 8). This indicated a change of flesh color from red to orange-yellow. These changes in $h^{\circ}$ value indicate over-ripening and senescence of watermelons which are subjected to prolonged storage (Kyriacou and Soteriou, 2015). In CR cultivar, effect of rootstocks on flesh color $h^{\circ}$ value was not significant after 21 days of storage, but control fruit had higher flesh color $h^{\circ}$ values than grafted fruit during shelf life (Table 7). In CT cultivar, fruit grafted on Macis and Argentario rootstocks and control fruit had higher flesh color $h^{\circ}$ values than 
Table 7. The effects of rootstocks on fruit flesh color $\left(L^{*}, C^{*}\right.$ and $\left.h^{\circ}\right)$, total lycopene $\left(\mu \mathrm{g} \mathrm{g}^{-1}\right)$ and beta carotene $\left(\mu \mathrm{g} \mathrm{g} \mathrm{g}^{-1}\right)$ of Crisby (CR) watermelon fruits during storage at $0^{\circ} \mathrm{C}$ and following 7 days at $21^{\circ} \mathrm{C}$

\begin{tabular}{|c|c|c|c|c|c|c|c|c|c|}
\hline \multirow{2}{*}{ Parameters } & \multirow{2}{*}{ Scion/ rootstock } & \multicolumn{3}{|c|}{ Days in storage at $0^{\circ} \mathrm{C}$} & & \multicolumn{3}{|c|}{ Days in shelf life at $21^{\circ} \mathrm{C}$} & \multirow{2}{*}{ Mean } \\
\hline & & $\begin{array}{ll}0 & 7 \\
\end{array}$ & 14 & 21 & Mean $0+7$ & $7+7$ & $14+7$ & $21+7$ & \\
\hline \multirow{5}{*}{$L^{*}$} & CR(Control) & 38.16 a 42.98 a & $44.94 \mathrm{a}$ & $43.87 a$ & 42.49 a $42.83 \mathrm{a}$ & $41.91 \mathrm{a}$ & a $43.52 \mathrm{a}$ & $45.39 \mathrm{a}$ & $43.41 \mathrm{a}$ \\
\hline & CR/Macis & 36.84 a 42.28 a & $44.01 \mathrm{a}$ & $44.77 a$ & 41.97 a $41.56 \mathrm{a}$ & $40.57 a$ & $42.64 \mathrm{a}$ & $42.04 \mathrm{a}$ & \\
\hline & CR/Argentario & 7.07 a 44.02 a & $43.16 \mathrm{a}$ & 43.12 a & 41.84 a $44.02 \mathrm{a}$ & $40.03 a$ & a $43.24 \mathrm{a}$ & $42.76 \mathrm{a}$ & \\
\hline & & $9.13 \mathrm{a} 42.32 \mathrm{a} a$ & $41.45 \mathrm{a}$ & 41.84 a & a $41.10 \mathrm{a}$ & $41.86 a$ & $42.14 \mathrm{a}$ & $42.14 \mathrm{a}$ & \\
\hline & $\mathrm{CR} / \mathrm{F}$ & 3.37 a 41.88 a & $42.37 \mathrm{a}$ & $44.99 a$ & a 41.48 a & $40.16 a$ & $42.60 \mathrm{a}$ & $43.67 \mathrm{a}$ & \\
\hline \multirow{5}{*}{$\mathrm{C}^{*}$} & & 3.32 a $31.31 \mathrm{~b}$ & $28.72 \mathrm{~d}$ & $28.71 \mathrm{k}$ & b $32.75 b$ & $34.51 \mathrm{a}$ & $29.40 \mathrm{~cd}$ & $29.15 b$ & \\
\hline & & 3.88 a 31.49 b & $32.94 \mathrm{c}$ & $29.36 \mathrm{k}$ & b 33.32 b & 36.42 & $29.11 \mathrm{~d}$ & $31.11 \mathrm{a}$ & \\
\hline & & 28.19 a $31.71 b$ & $32.52 \mathrm{~b}$ & $30.41 \mathrm{~b}$ & b 32.58 b & $35.22 \mathrm{a}$ & a $30.60 \mathrm{bc}$ & $32.18 \mathrm{a}$ & \\
\hline & & 9.10 a 37.09 a & $35.77 \mathrm{a}$ & 34.58 & a 35.76 a & $35.67 a$ & $33.61 \mathrm{a}$ & $32.27 \mathrm{a}$ & \\
\hline & & .32 a 35.81 a & $34.72 b$ & 30.86 & a 35.86 a & $34.56 \mathrm{a}$ & $32.66 \mathrm{ab}$ & $32.08 \mathrm{a}$ & \\
\hline \multirow{5}{*}{$h^{\circ}$} & & 3.17 a 45.76 a & $46.28 \mathrm{ab}$ & 48.22 & $44.08 \mathrm{a}$ & 45.87 & $47.49 \mathrm{a}$ & $47.44 \mathrm{a}$ & \\
\hline & & 5.86 b $45.59 a$ & $47.35 \mathrm{a}$ & 46.55 & a 44.27 a & $44.59 a$ & $46.10 \mathrm{a}$ & $45.15 b c$ & \\
\hline & tario & $6.76 \mathrm{~b} 44.37 \mathrm{a}$ & $46.91 \mathrm{a}$ & 47.37 a & 43.85 a $44.58 \mathrm{a}$ & $43.43 a$ & 45.28 a & $45.85 a b$ & $a b$ \\
\hline & & 5.47 b 45.05 a & $45.60 \mathrm{~b}$ & 47.13 & $1 \mathrm{a} 42.72 \mathrm{~b}$ & $43.80 \mathrm{a}$ & $45.33 \mathrm{a}$ & $43.58 \mathrm{c}$ & \\
\hline & & $5.92 \mathrm{~b} 44.05 \mathrm{a}$ & $45.26 \mathrm{~b}$ & $47.22 \mathrm{a}$ & $\mathrm{a} 42.41 \mathrm{~b}$ & $42.87 \mathrm{a}$ & $44.84 \mathrm{a}$ & $45.12 \mathrm{bc}$ & \\
\hline \multirow{5}{*}{$\begin{array}{l}\text { Lycopene } \\
\left(\mu g^{-1}\right)\end{array}$} & & .38 a 37.26 a & $28.32 b$ & 23.01 & a 27.84 c & 38.88 & $23.55 \mathrm{c}$ & $20.49 a$ & \\
\hline & & .25 a 43.90 a & $27.42 b$ & 22.50 & a 30.23 bc & 38.13 & $24.65 \mathrm{c}$ & $20.98 a$ & \\
\hline & & 40.52 a 35.16 a & $26.84 \mathrm{~b}$ & 24.59 & 8 a 31.31 bc & 34.91 & $26.48 \mathrm{bc}$ & $17.85 \mathrm{a}$ & $4 \mathrm{~b}$ \\
\hline & & 43.02 a 38.27 a & $32.67 \mathrm{a}$ & 28.21 & 4 a 38.88 a & $44.65 a$ & 33.62 a & $20.14 \mathrm{a}$ & $2 a$ \\
\hline & & 42.10 a $36.63 a$ & $32.38 \mathrm{a}$ & 28.64 & $4 a 34.38 a b$ & $41.81 \mathrm{a}$ & $30.49 \mathrm{ab}$ & $18.95 \mathrm{a}$ & 31 \\
\hline \multirow{5}{*}{$\begin{array}{l}\text { Beta } \\
\text { carotene } \\
\left(\mu \mathrm{g} \mathrm{g}^{-1}\right)\end{array}$} & & $17 \mathrm{a} \quad 0.14 \mathrm{a}$ & $0.18 \mathrm{a}$ & $0.12 \mathrm{a}$ & a $0.21 \mathrm{a}$ & $0.18 \mathrm{a}$ & $0.18 \mathrm{a}$ & $0.18 \mathrm{a}$ & \\
\hline & & $0.11 \mathrm{a}$ & $0.18 \mathrm{a}$ & $0.12 \mathrm{a}$ & $0.14 \mathrm{a} \quad 0.21 \mathrm{a}$ & $0.19 a$ & $0.15 \mathrm{a}$ & $0.16 \mathrm{a}$ & \\
\hline & CR/RS841 & $0.15 a$ & $0.15 \mathrm{a}$ & $0.12 \mathrm{a}$ & 0.15 a $\quad 0.24 a$ & $0.16 \mathrm{a}$ & $0.14 \mathrm{a}$ & $0.10 \mathrm{a}$ & $0.16 \mathrm{a}$ \\
\hline & $\mathrm{CR} /$ Argentario & $0.23 \mathrm{a} \quad 0.21 \mathrm{a}$ & $0.18 \mathrm{a}$ & $0.11 \mathrm{a}$ & 0.18 a $\quad 0.28 a$ & $0.22 \mathrm{a}$ & $0.15 \mathrm{a}$ & $0.15 \mathrm{a}$ & $0.20 \mathrm{a}$ \\
\hline & CR/Ferro & $0.17 \mathrm{a} \quad 0.16 \mathrm{a}$ & $0.18 \mathrm{a}$ & $0.15 \mathrm{a}$ & 0.17 a $\quad 0.33 a$ & $0.20 \mathrm{a}$ & $0.20 \mathrm{a}$ & $0.18 \mathrm{a}$ & $0.23 \mathrm{a}$ \\
\hline
\end{tabular}

${ }^{x}$ Mean separation was performed by Fisher's LSD test. Means $(n=3)$ followed by same letters within a column are not significantly different at $\mathrm{P}<0.05$.

Table 8. The effects of rootstocks on fruit flesh color $\left(L^{*}, C^{*}\right.$ and $\left.h^{\circ}\right)$, total lycopene $\left(\mu \mathrm{g} \mathrm{g}^{-1}\right)$ and beta carotene $\left(\mu \mathrm{g} \mathrm{g} \mathrm{g}^{-1}\right)$ of Crimson Tide (CT) watermelon fruits during storage at $0^{\circ} \mathrm{C}$ and following 7 days at $21^{\circ} \mathrm{C}$

\begin{tabular}{|c|c|c|c|c|c|c|c|c|c|}
\hline \multirow{2}{*}{ Parameters } & \multirow{2}{*}{ Scion/ rootstock } & \multicolumn{3}{|c|}{ Days in storage at $0^{\circ} \mathrm{C}$} & \multirow{2}{*}{ Mean } & \multicolumn{3}{|c|}{ Days in shelf life at $21^{\circ} \mathrm{C}$} & \multirow{2}{*}{ Mean } \\
\hline & & 7 & 14 & 21 & & $0+7$ & $7+7$ & $14+7 \quad 21+7$ & \\
\hline \multirow{5}{*}{$L^{*}$} & CT(Control) & 40.46 a $41.85 a$ & $43.09 \mathrm{a}$ & $45.35 \mathrm{a}$ & $42.69 \mathrm{a}$ & $45.55 \mathrm{a}$ & $43.57 \mathrm{a}$ & 29.06 a 40.76 a & $39.73 \mathrm{a}$ \\
\hline & & $43.08 \mathrm{a} 44.15 \mathrm{a}$ & $42.10 \mathrm{a}$ & $44.82 \mathrm{a}$ & & $44.36 \mathrm{a}$ & $42.48 \mathrm{a}$ & $27.37 \mathrm{a} 41.35 \mathrm{a}$ & \\
\hline & CT/Argentario & $40.73 \mathrm{a} 43.11 \mathrm{a}$ & $42.36 \mathrm{a}$ & $46.94 \mathrm{a}$ & $43.29 \mathrm{a}$ & $40.83 \mathrm{a}$ & $41.65 \mathrm{a}$ & 27.89 a 43.35 a & \\
\hline & $\mathrm{CT} / \mathrm{R}$ & $43.91 \mathrm{a} 42.65 \mathrm{a}$ & $42.10 \mathrm{a}$ & $46.21 \mathrm{a}$ & $43.72 \mathrm{a}$ & $41.52 \mathrm{a}$ & $40.64 \mathrm{a}$ & 30.94 a 45.71 a & $39.71 \mathrm{a}$ \\
\hline & & 39.17 a 42.03 a & $43.92 \mathrm{a}$ & $43.85 \mathrm{a}$ & $42.24 \mathrm{a}$ & $41.60 \mathrm{a}$ & $43.38 \mathrm{a}$ & 30.21 a $45.51 \mathrm{a}$ & $7 \mathrm{a}$ \\
\hline \multirow{5}{*}{$\mathrm{C}^{*}$} & & .91 b $32.95 a$ & $31.29 \mathrm{c}$ & $30.99 \mathrm{~b}$ & $C$ & $35.27 \mathrm{c}$ & $32.19 \mathrm{c}$ & 16.99 a $25.02 b$ & $7 \mathrm{a}$ \\
\hline & & 33.99 b34.01 a & $34.71 \mathrm{~b}$ & $31.84 \mathrm{~b}$ & & $37.01 \mathrm{~b}$ & $33.98 \mathrm{bc}$ & 18.76 a 26.92 b & $7 \mathrm{a}$ \\
\hline & & 37.07 a $33.76 \mathrm{a}$ & $34.63 \mathrm{~b}$ & $34.29 \mathrm{a}$ & & $39.74 \mathrm{a}$ & $38.01 \mathrm{a}$ & 18.49 a 30.81 a & $6 a$ \\
\hline & & 36.25 a 34.93 a & $36.55 a b$ & $34.60 a$ & & $39.10 \mathrm{a}$ & $33.82 \mathrm{bc}$ & 17.69 a 31.39 a & \\
\hline & & 37.35 a 34.27 a & $37.76 \mathrm{a}$ & $35.27 \mathrm{a}$ & $36.16 \mathrm{a}$ & $36.98 \mathrm{a}$ & $35.40 \mathrm{~b}$ & 17.38 a $33.05 a$ & $30.70 \mathrm{a}$ \\
\hline \multirow{5}{*}{$h^{\circ}$} & & $.61 \mathrm{a} 42.50 \mathrm{a}$ & $44.37 a b$ & $46.49 a$ & $43.24 \mathrm{a}$ & $45.22 \mathrm{a}$ & $43.65 a b$ & $44.48 \mathrm{a} 45.42 \mathrm{a}$ & $9 \mathrm{a}$ \\
\hline & & 9 a 42.40 al & $44.52 \mathrm{a}$ & $46.61 \mathrm{a}$ & & $43.77 \mathrm{ab}$ & $44.48 \mathrm{a}$ & 42.48 a $44.33 a$ & $6 \mathrm{ab}$ \\
\hline & & $40.29 a 41.78 b$ & $43.08 \mathrm{c}$ & $46.45 \mathrm{a}$ & $42.90 \mathrm{al}$ & $42.72 b$ & $44.22 a b$ & 43.00 a $45.52 \mathrm{a}$ & $43.86 a b$ \\
\hline & & 40.63 a $41.03 \mathrm{c}$ & $43.08 \mathrm{c}$ & $44.68 \mathrm{ab}$ & & $42.41 \mathrm{~b}$ & $40.67 \mathrm{c}$ & $42.36 \mathrm{a} 43.64 \mathrm{a}$ & 42. \\
\hline & & 39.75 a $40.76 c$ & $43.20 \mathrm{bc}$ & $43.88 \mathrm{~b}$ & & $41.84 \mathrm{~b}$ & $41.95 \mathrm{~b} \mathrm{c}$ & 44.54 a $44.37 \mathrm{a}$ & 43 \\
\hline \multirow{5}{*}{$\begin{array}{l}\text { Lycopene } \\
\left(\mu \mathrm{g} \mathrm{g}^{-1}\right)\end{array}$} & & .53 c 30.03 c & $25.77 \mathrm{c}$ & $31.37 \mathrm{~b}$ & & $35.41 \mathrm{c}$ & $32.50 \mathrm{a}$ & 31.06 b 35.74 b & \\
\hline & & 33.08 c $30.00 \mathrm{c}$ & $32.18 \mathrm{bc}$ & $24.63 c$ & $29.97 b$ & $35.27 \mathrm{c}$ & $32.49 \mathrm{a}$ & 36.70 b 35.48 b & $34.98 \mathrm{c}$ \\
\hline & ntario & 55.10 a 52.68 a & $36.02 \mathrm{ab}$ & $32.89 a b$ & & $42.99 \mathrm{ab}$ & $34.64 \mathrm{a}$ & 35.62 b $39.10 a b$ & $38.09 \mathrm{~b}$ \\
\hline & & $45.05 \mathrm{~b} 43.25 \mathrm{~b}$ & $44.02 \mathrm{a}$ & $36.92 \mathrm{ab}$ & & $40.11 b$ & $36.21 \mathrm{a}$ & $45.00 \mathrm{a} 41.74 \mathrm{a}$ & $40.76 \mathrm{a}$ \\
\hline & & $46.89 \mathrm{~b} 44.15 \mathrm{~b}$ & $41.42 \mathrm{a}$ & $38.69 \mathrm{a}$ & 42.7 & $46.00 \mathrm{a}$ & $39.44 \mathrm{a}$ & $45.16 \mathrm{a} 43.12 \mathrm{a}$ & 43. \\
\hline \multirow{5}{*}{$\begin{array}{l}\text { Beta } \\
\text { carotene } \\
\left(\mu \mathrm{g} \mathrm{g}^{-1}\right)\end{array}$} & & 16 a $0.12 \mathrm{a}$ & $0.18 \mathrm{a}$ & $0.11 \mathrm{a}$ & & $0.15 \mathrm{a}$ & & $0.11 \mathrm{a} \quad 0.10 \mathrm{a}$ & $2 a$ \\
\hline & & $0.12 \mathrm{a}$ & $0.13 \mathrm{a}$ & $0.14 \mathrm{a}$ & & $0.17 \mathrm{a}$ & $0.09 \mathrm{a}$ & $0.16 \mathrm{a}$ & \\
\hline & CT/Argentario & $0.19 \mathrm{a}$ & $0.14 \mathrm{a}$ & $0.11 \mathrm{a}$ & $0.15 a$ & $0.12 \mathrm{a}$ & $0.17 \mathrm{a}$ & $0.09 a$ & $0.13 \mathrm{a}$ \\
\hline & CT/RS841 & $0.30 \mathrm{a} \quad 0.09 \mathrm{a}$ & $0.06 \mathrm{a}$ & $0.08 \mathrm{a}$ & $0.13 \mathrm{a}$ & $0.14 \mathrm{a}$ & $0.10 \mathrm{a}$ & $0.10 \mathrm{a}$ & $0.10 \mathrm{a}$ \\
\hline & CT/Ferro & 0.16 a $\quad 0.14 a$ & $0.11 \mathrm{a}$ & $0.06 \mathrm{a}$ & $0.12 \mathrm{a}$ & $0.11 \mathrm{a}$ & $0.06 \mathrm{a}$ & $0.13 a \quad 0.10 a$ & $0.10 \mathrm{a}$ \\
\hline
\end{tabular}


other grafted fruit during storage and shelf life (Table 8). Flesh color changes were observed in the fruits, suggesting that fruit ripening occurs faster in control fruits than grafted fruit during storage. Özdemir et al. (2018) reported similar findings with the fruit from grafted watermelons. Watermelon flesh color varies from brilliant red (poor flesh color $h^{\circ}$ ) to orange red (top flesh color $h^{\circ}$ ) as ripening level progresses. Özdemir et al. (2016) reported that grafted and non-grafted fruit showed a progressive increase in flesh color $\mathrm{h}^{\circ}$ value after shelf life period following storage, indicating a shift from red to orange-yellow. This changes in flesh color $h^{\circ}$ value, characteristic of over-ripening and senescence has been reported after prolonged postharvest storage of watermelons (Kyriacou and Soteriou, 2015).

Lycopene content in both cultivars showed similar trend with flesh color $\mathrm{C}^{*}$ values (Table 7 and 8). Lycopene content significantly decreased at the end of storage for both cultivars. In CR cultivar, effect of rootstocks on lycopene content was not significant after 21 days of storage, but fruit grafted on RS841 and Ferro rootstocks had higher lycopene content than grafted fruit during shelf life (Table 7).

In CT cultivar, fruit grafted on RS841, Argentario and Ferro rootstocks had higher lycopene content than those on Macis rootstock and control fruit after 21 days of storage and fruit grafted on RS841 and Ferro rootstocks had higher lycopene content than those other rootstock and control fruit during shelf life (Table 8). It was reported that grafted plants higher lycopene content than non-grafted watermelon fruit during storage. (Kyriacou and Soteriou, 2015). The overall intensity of flesh color $\left(C^{*}\right.$ value), hue angle $\left(h^{\circ}\right.$ value) and lycopene content were impressed by storage time and rootstocks (Özdemir et al., 2016). The increase in flesh color $C^{*}$ value of watermelon fruit was probably as a result of the increase in lycopene content (Perkins-Veazie and Collins, 2006). Postharvest color changes and lycopene biosynthesis in watermelons can be affected by storage temperature and cultivar (Özdemir et al., 2018). Perkins-Veazie and Collins (2006) reported that watermelons stored at $21^{\circ} \mathrm{C}$ had higher flesh color $\mathrm{C}^{*}$ value and lycopene content, compared to initial value at harvest whereas no or little change was observed in flesh color $C^{*}$ value and lycopene content of fruit held at $5^{\circ} \mathrm{C}$ or $13^{\circ} \mathrm{C}$ depending on cultivars. Degradation in lycopene during senescence of non-grafted watermelon fruit and grafted fruit after prolonged storage and consequent shelf life period led to decrease in flesh color $\mathrm{C}^{*}$ value and increase in flesh color $h^{\circ}$ value (Özdemir et al., 2018).

Effects of grafting on $\beta$-carotene content were not significant at the end of the storage time and shelf life for both cultivars (Table 7 and 8). Özdemir et al. $(2016,2018)$ reported similar findings with the fruit from grafted watermelons. Perkins-Veazie and
Collins (2006) reported that watermelons stored for 14 days at $21^{\circ} \mathrm{C}$ gained $50-139 \%$ in $\beta$-carotene compared to fresh fruit, whereas fruit held at 5 and $13^{\circ} \mathrm{C}$ changed little in $\beta$-carotene content. In our study, $\beta$-carotene content decrease during storage and shelf life.

\section{Conclusions}

The $\mathrm{Cl}$ areas covered $<25 \%$ of rind surface of fruit for both cultivars. The effect of rootstocks and control fruit on the incidence of rind and flesh $\mathrm{Cl}$ was not significant after 21 days of storage and shelf life in CR and CT cultivars. Weight loss in grafted and control fruit were very low $(<1 \%)$ during storage for both cultivars. Fungal decay was not observed during storage for both cultivars. However, it was observed during the shelf life.

The decayed areas covered $<10 \%$ of rind surface of fruit. The graft combinations did not differ in the incidence of fungal during shelf life for both cultivars. TSS content remained above $10 \%$ in fruit of both cultivars throughout storage period. TA content slightly increased in parallel with changes in juice $\mathrm{pH}$ during storage and shelf life for both cultivars at the end of the storage time and additional 7 days shelf life at $21^{\circ} \mathrm{C}$. The citric acid content from organic acids changed from $0.06 \%$ to $0.09 \%$ for CR cultivar and $0.06 \%$ to $0.10 \%$ for CT cultivar and the malic acid content changed from $0.19 \%$ to $0.25 \%$ for CR cultivar and $0.21 \%$ to $0.32 \%$ for CT cultivar after 21 days of storage and shelf life. The effects of grafting on hallow heart were not significant during the storage and shelf life for both cultivars. The most abundant sugar was sucrose at the end of the storage time and shelf life in both cultivars.

Taste scores (1-9) declined to the lowest level for 21 days of storage at $0^{\circ} \mathrm{C}$ in $\mathrm{CR}$ cultivar and the lowest level during shelf life in CT cultivar. Effects of grafting on flesh color lightness ( $L^{*}$ value) was not significant at the end of the storage time and shelf life for both cultivars. The flesh color $h^{\circ}$ values showed a progressive increase in non-grafted fruit with a lesser extent in grafted fruit during storage in both cultivars. Lycopene content significantly decreased at the end of storage for both cultivars.

Effects of grafting on $\beta$-carotene content were not significant during the storage at $0^{\circ} \mathrm{C}$ for 21 days and additional 7 days shelf life at $21^{\circ} \mathrm{C}$ for both cultivars. During the storage and shelf life, watermelons grafted on Ferro and RS841 rootstocks retained fruit flesh firmness, compared to the non-grafted fruit for both cultivars.

Watermelons grafted on Ferro and RS841 rootstocks had higher flesh color with lower ripening and softening and higher lycopene content for CR and/or CT fruit during shelf life. Taste scores of grafted fruit had scored higher than control fruits. Watermelons could be kept for 7 days at $0{ }^{\circ} \mathrm{C}$ without rind and flesh $\mathrm{Cl}$. 


\section{Acknowledgments}

The Scientific and Technological Research Council of Turkey was supported this research (TUBITAK $108 \mathrm{O}$ 391). The authors wish to thank the TUBITAK for support.

\section{References}

Araújo Neto, S.E., Hafle, O.M., Gurgel, F.L., Menezes, J.B., \& Silva, G.G. (2000). Quality and postharvest shelf life of crimson sweet watermelon marketed in Mossoro. Revista Brasileira de Engenharia Agrícola e Ambiental, 4:235-239.

Bruton, B.D., Fish, W.W., Roberts, W., \& Popham, T.W. (2009). The influence of rootstock selection on fruit quality attributes of watermelon. The Open Food Science Journal, 3:15-34.

Chisholm, D.N., \& Picha, D.H. (1986). Effect of storage temperature on sugar and organic acid contents of watermelons. HortScience, 21:1031-1033.

Cushman, K.E., \& Huan, J. (2008). Performance of four triploid watermelon cultivars grafted onto five rootstock genotypes: Yield and fruit quality under commercial growing conditions. Acta Horticulturae, 782:335-342.

Çandır, E., Yetişir, H., Karaca, F., \& Üstün, D. (2013). Phytochemical characteristics of grafted watermelon on different bottle gourds (Lagenaria siceraria) collected from the Mediterranean region of Turkey. Turkish Journal of Agriculture and Forestry, 37:443456.

Davis, A.R., \& Perkins-Veazie, P. (2005). Rootstock effects on plant vigor and watermelon fruit quality. Cucurbit Genetics Cooperative Report, 28:39-42.

Davis, A.R., Perkins-Veazie, P., Sakata, Y., LopezGalarza, S., Maroto, J.V., Lee, S.G., Huh, Y.C., Sun, Z., Miguel, A., King, S.R., Cohen, R., \& Lee, J.M. (2008). Cucurbit grafting. Critical Reviews in Plant Sciences, 27:50-74.

Karaağaç, O., Balkaya, A., \& Kafkas, N.E. (2018). Effect of rootstocks on fruit quality and aroma characteristics of watermelon (Citrullus lanatus). Anadolu Journal of Agricultural Science, 33:92-104.

Kyriacou, M.C., \& Soteriou, G. (2015). Quality and postharvest performance of watermelon fruit in response to grafting on interspecific cucurbit rootstocks. Journal of Food Quality, 38:21-29.

Lee, J.M., \& Oda, M. (2003). Grafting of herbaceous vegetable and ornamental crops. Horticultural Review, 28:61-124.

Liu, G.N., Zhu, Y.H., \& Jiang, J.G. (2009). The metabolomic of carotenoids in engineered cell factory. Applied Microbiology and Biotechnology, 83:989-999.

McGuire, R.G. (1992). Reporting of objective colour measurement. Hort-Science, 27:1254-1255.

Miguel, A., Maroto, J.V., San Bautista, A., Baixauli, C., Cebolla, V., Pascual, B., Lopez, S., \& Guardiola, J.L. (2004). The grafting of triploid watermelon is an advantageous alternative to soil fumigation by methyl bromide for control of Fusarium wilt. Scientia Horticulturae, 103:9-17.
Özdemir, A.E., Candır, E., Yetişir, H., Aras, V., Arslan, Ö., Baltaer, Ö., Üstün, D., \& Ünlü, M. (2016). Effects rootstocks on storage and shelf life of grafted watermelons. Journal of Applied Botany and Food Quality, 89:191-201.

Özdemir, A.E., Candır, E., Yetişir, H., Aras, V., Arslan, Ö., Baltaer, Ö., Üstün, D., \& Ünlü, M. (2018). Rootstocks affected postharvest performance of grafted Crisby and Crimson Tide watermelon cultivars. Journal of Agricultural Sciences, 24:453-462.

Perkins-Veazie, P., \& Collins, J.K. (2006). Carotenoid changes of intact watermelons after storage. Journal of Agricultural and Food Chemistry, 54:5868-5874.

Picha, D.H. (1986). Postharvest fruit conditioning reduces chilling injury in watermelons. HortScience, 21:14071409.

Proietti, S., Rouphael, Y., Colla, G., Cardarelli, M., De Agazio, M., Zacchini, M., Rea, E., Moscatello, S., \& Battistelli, A. (2008). Fruit quality of mini-watermelon as affected by grafting and irrigation regimes. Journal of The Science of Food and Agriculture, 88:11071114

Radulovic, M., Ban, D., Sladonja, B., \& Lusetic-Bursic, V. (2007). Changes of quality parameters in watermelon during storage. Acta Horticulturae, 731:451-456.

Risse, L.A., Brecht, J.K., Sargent, S.A., Locascio, S.J., Crall, J.M., Elmstrom, G.W., \& Maynard, D.N. (1990). Storage characteristics of small watermelon cultivars. Journal of The American Society for Horticultural Science, 115:440-443.

Rushing, J.W., Fonseca, J.M., \& Keinath, A.P. (2001). Harvesting and post-harvest handling. In: Watermelons-Characteristics, Production, and Marketing, (Ed: D. N., Maynard), Alexandria, VA, American Society for Horticultural Science Press, pp: $156-164$.

SAS. (2019). SAS/STAT User's Guide, Version 9.4. SAS Institute Inc., NC, USA.

Soteriou, G.A., Kyriacou, M.C., Siomos, A.S., \& Gerasopoulos, D. (2014). Evolution of watermelon fruit physicochemical and phytochemical composition during ripening as affected by grafting. Food Chemistry, 165:282-289.

Suárez-Hernández, Á.M., Grimaldo-Juárez, O., GarcíaLópez, A.M., González-Mendoza, D., \& HuitrónRamírez, M.V. (2016). Influence of rootstock on postharvest watermelon quality. Revista Chapingo Serie Horticultura, 23:49-58.

Suslow, T.V. (1997). Watermelon: Recommendations for maintaining postharvest quality. http://postharvest.ucdavis.edu/Commodity_Resource s/Fact_Sheets/Datastores/Fruit_English/?uid=60\&ds $=798$ (Accessed: 27 July, 2020).

Taylor, M., Bruton, B.D., Fish, W.W., \& Roberts, W. (2006). Cost benefit analyses of using grafted watermelons for disease control and the fresh-cut market. In: Cucurbitaceae 2006, Asheville, North Carolina, 277-285.

Yetişir, H., Sarı, N., \& Yücel, S. (2003). Rootstock resistance to Fusarium wilt and effect on watermelon fruit yield and quality. Phytoparasitica, 31:163-169. 